\title{
Trajectory of Post-traumatic Stress and Depression among Children and Adolescents Following Single-incident trauma
}

Joyce Zhang ${ }^{\mathrm{a}}$, Richard Meiser-Stedman ${ }^{\mathrm{a}}$, Bobby Jones ${ }^{\mathrm{b}}$, Patrick Smith ${ }^{\mathrm{c}}$, Tim Dalgleish $^{\mathrm{d}, \mathrm{j}}$, Adrian Boyle ${ }^{\mathrm{e}}$, Clare Dixon ${ }^{\mathrm{f}}$, Lysandra Sinclaire-Harding ${ }^{\mathrm{g}}$, Susanne Schweizer ${ }^{\mathrm{i}}$, Jill Newby ${ }^{\mathrm{h}}$, Anna McKinnon ${ }^{\mathrm{j}}$

${ }^{a}$ Department of Clinical Psychology, University of East Anglia, UK; ${ }^{b}$ Department of Psychiatry, University of Pittsburgh, United States; 'Department of Psychology, Institute of Psychiatry, Psychology and Neuroscience, King's College, London, UK; ${ }^{d}$ Medical Research Council Cognition and Brain Sciences Unit, University of Cambridge, UK; ${ }^{j}$ Cambridgeshire and Peterborough NHS Foundation Trust, UK; ${ }^{e}$ Emergency Department, Addenbrooke's

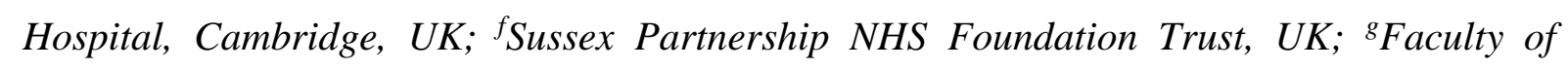
Education University of Cambridge, UK; ${ }^{h}$ School of Psychiatry, University of New South Wales, Australia; ${ }^{i}$ School of Psychology, University of New South Wales, Australia; ${ }^{j}$ Department of Psychology, Macquarie University, Australia.

Corresponding author: Joyce Zhang, Department of Clinical Psychology, University of East Anglia, Norwich Medical School, Norwich Research Park, NR4 7TJ, Norwich, UK; Email: y.zhang41@uea.ac.uk

Acknowledgements: This research received no specific grant from any funding agency, commercial or not-for-profit sectors. The authors have declared that they have no competing or potential conflicts of interest. 


\title{
Trajectory of Post-traumatic Stress and Depression among Children and Adolescents Following Single-incident trauma: Courses and Risks
}

\begin{abstract}
Objective: PTSD-depression has high comorbidity and understanding their relationship is of clinical and theoretical importance. A comprehensive way to understand posttrauma psychopathology is through symptom trajectories. The study aimed to look at the developmental courses of PTSD and depression symptoms in the initial months posttrauma, and their interrelationship in children and adolescents by utilizing advanced group-based trajectory modeling (GBTM). Methods: Two-hundred-and-seventeen children and adolescents aged between eight and 17 exposed to single-event trauma were included in the study. Their PTSD and depression symptoms were measured at two weeks, two months and nine months, with further psychological variables measures at the two-week assessment. Results: The GBTM modeling yielded a three-group model for PTSD and a three-group model for depression. All participants' PTSD symptoms reduced to non-clinical level by nine months: participants were observed to be resilient $(42.4 \%)$ or to be able to recover within two months $(35.6 \%)$, while $21.9 \%$ experienced high level PTSD symptoms but recovered in nine months time. The depression trajectories described a chronic non-recovery depression group (20.1\%) and two mild symptom groups $(45.9 \%, 34.0 \%)$. Further analysis showed high synchronicity between PTSS and depression groups but predictor analysis revealed disparate predictors. Perievent panic (CPP), appraisal (CPTCI), rumination and thought suppression at week two predicted slow recovery from PTSS, while pre-trauma wellbeing (CPAS), post-trauma anxiety (SCAS) and appraisal (CPTCI) predicted chronic depression. Conclusions: Posttrauma depression was more persisting than PTSD symptoms at nine months in the sampled population. Cognitive appraisal may be central to explaining PTSD-depression comorbidity.
\end{abstract}


Keywords: PTSD; depression; comorbidity; trajectory; GBTM; risk factors; children; adolescents 


\section{Introduction}

The co-occurrence of posttraumatic stress disorder (PTSD) and depression has been widely observed. In their meta-analysis, Rytwinski, Scur, Feeny, and Youngstrom (2013) reported that the prevalence of PTSD and major depression disorder comorbidity was 52\% (95\% CI [48, 56]) in adults. A recent meta-analysis estimated the prevalence of depression to be $24.2 \%$ (95\% CI [20.6-28.0]) in trauma-exposed children and adolescents, and estimated the odds ratio of having a depression diagnosis to be 2.6 (95\% CI [2.0,3.3]) for those exposed to trauma, compared with no or only mild trauma exposure (Vibhakar et al., 2019).

PTSD-depression comorbidity is known for being associated with more severe impairments in various domains including biology, affect regulation, dissociation, behavioral control, cognition and self-concept (Clark \& Kirisci, 1996; Cook et al., 2017; Lai, La Greca, Auslander, \& Short, 2013; Mazza \& Reynolds, 1999). Many studies have been conducted to investigate the mechanism of how PTSD and depression interact. The past 20 years have seen inconsistent, sometimes contradictory, findings (Bleich, Koslowsky, Dolev, \& Lerer, 1997; Breslau, Davis, Peterson, \& Schultz, 2000; Jovanovic et al., 2010; Shalev et al., 1998; Spinhoven, Penninx, van Hemert, de Rooij, \& Elzinga, 2014; Thabet, Abed, \& Vostanis, 2004). A systematic review has outlined several hypotheses that are better supported with evidence in adult studies (Stander, Thomsen, \& Highfill-McRoy, 2014). In brief, these hypotheses are: the effects of PTSD on the development of depression are stronger than the reverse risk; PTSD and depression share common risk factors; they are not completely independent diagnoses nor are they two aspects of a same spectrum of traumatic response. Although no systematic review on the relation between PTSD and depression in children and adolescents has been conducted, it is plausible to postulate that the above hypotheses may still stand since there is no fundamental distinction in the theoretical framework of PTSD and depression between adults 
and the younger population. As few studies have thoroughly examined the connection relating to children and adolescence, the present study aims to address the question.

One of the challenges in PTSD is to define what represents a typical or an atypical response to trauma. As acute disturbances in mood, cognition and behavior are considered normal in responding to a trauma and as there are late onset cases where symptoms stay dormant for months or even years after the trauma, the interpretation of posttraumatic symptoms will only be meaningful when levels of severity are put onto a time dimension. In fact, following the prototypical patterns across time (resilient, recovery, delayed and chronic) proposed by Bonanno (2004), posttraumatic trajectory has become a research interest. A commonly observed PTSD trajectory is that, despite of the diversity in the nature of the trauma, elevated symptoms do not last longer than six months and they are often followed by a spontaneous recovery (Fan et al., 2015; Hiller et al., 2016; Le Brocque, Hendrikz \& Kenardy, 2009; Maikovich, Koenen \& Jaffee, 2009; Osofsky, Osofsky, Weems, King \& Hansel, 2015; Punamäki, Palosaari, Diab, Peltonen \& Qouta, 2015; Santiago et al., 2013; Wang, Chan \& Ho, 2013), so that early intervention may not be beneficial (Kearns, Ressler, Zatzick, \& Rothbaum, 2012; Litz, Gray, Bryant, \& Adler, 2002; Stallard et al., 2006). Meanwhile, what is a representative post-trauma depression trajectory remains unclear due to limited research. Therefore, we argue that a comprehensive way to study the PTSD-depression comorbidity is to examine the PTSD and depression trajectory simultaneously. The approach gives a few advantages. First, it will provide more empirical data about the much-desired post-trauma depression trajectory. Secondly, how the symptoms evolve could reveal their relationship. High synchronicity between them (e.g., chronic PTSD trajectory accompanied by chronic depression course) may suggest that the two share a linked mechanism; otherwise, they should be viewed as independent responses to trauma. 
Although trajectory provides an in-depth angle to study PTSD, the conventional methods (e.g., averaging, classifying on subjective criteria) to identify trajectory groups are inadequate (Bonanno \& Mancini, 2012). This has given rise to the rapidly growing application of statistical modelling that were devised to identify latent sub-populations. Nevertheless, there are yet few child and adolescent studies that have adopted this modelling approach.

In a review of post-trauma response trajectory, Galatzer-Levy, Huang \& Bonanno (2018) enlisted 54 modelling-based trajectory studies and only nine were from the youth literature. Two of these considered internalizing and externalizing problems in children from wareffected area (Betancourt, McBain, Newnham \& Brennan, 2013; Betancourt, McBain \& Brennan, 2014), one examined anxiety and depression among child survivors of maltreatment (Lauterbach \& Armour, 2016), and the remaining six focused on PTSD symptoms. Among those that addressed the PTSD trajectory, the nature of the trauma varied from natural disaster (La Greca et al., 2013; Self-Brown, Lai, Thompson, McGill \& Kelley, 2013), accidental injury (Hong et al., 2014; Le Brocque et al., 2009), family violence (Nugent et al., 2009) and war (Punamäki et al., 2015). Notably, Hong et al. (2014) examined the association between PTSD and depression. However, instead of applying trajectory modeling to both outcomes, they applied regression between depression symptoms and PTSD trajectory groups (recovery $19.9 \%$, resilience $72.7 \%$, chronic $1.8 \%$ and delayed reactions $5.6 \%$ ). They reported a significant correlation across all PTSD groups at the three time points except for resilience and delayed reactions groups at two days and six months, however the implication was very difficult to interpret. The study in the Galatzer-Levy et al. (2018) review that thoroughly addressed PTSD, depression and their relationship using a trajectory approach was a latent growth mixture modeling (LGMM) study in adult traumatic injury. De Roon-Cassini, Mancini, Rusch, \& Bonanno (2010) reported four PTSD (low symptom 59\%, chronic 22\%, delayed 6\% and recovering $13 \%$ ) trajectories and four matching depression groups. They also assessed the 
concordance of PTSD and depression classifications by chi-square and Haberman's adjusted residual (HAR) analysis and reported that $69.7 \%$ of the samples were assigned to the matched groups (e.g., low PTSD and low depression). Nevertheless, a direct link between the two outcomes was still missing, in the sense that neither chi-square nor HAS was able to give the probability that a sample would be classified to a depression group based on their PTSD group profile or vice versa.

The present study looks at the natural trajectories of PTSD and depression symptoms and their relation to each other in children and adolescents by utilizing group-based trajectory modelling (GBTM). GBTM, equivalent to latent class growth analysis (LCGA), has evinced solid performance in clinical research (Nagin \& Odgers, 2010; Twisk \& Hoekstra, 2012). Further, the joint trajectory model extension made it possible to analyze the connections between the trajectories of two outcomes (Jones \& Nagin, 2007). Specifically, it reports the conditional probabilities linking membership across the trajectory groups of the two respective symptoms.

Having meaningfully classified trajectory groups allows a study to identify potential predictors that are associated with resilience, recovery and pathology. By comparing the variety and magnitude of the factors predicting PTSD with those predicting depression, one may reveal valuable information for understanding the broader posttrauma responses in children and adolescents. The predictors chosen for the study are: age, gender, pre-trauma emotional wellbeing, peri-event panic, post-trauma anxiety, appraisal, adaptive processing, thought suppression, rumination and self-blame. These putative risk factors were selected for the following reasons. First, there have been conflicting reports on the prevalence of PTSD according to gender and age in the child/adolescent population (Breslau, Davis, Andreski, Peterson, \& Schultz, 1997; Contractor et al., 2013; Green et al., 1991; Thabet et al., 2004; Trickey, Siddaway, Meiser-Stedman, Serpell, \& Field, 2012), whereas depression tends to be 
more prevalent in older female adolescents (Allgood-Merten, Lewinsohn, \& Hops, 1990; Saluja et al., 2004; Thapar, Collishaw, Pine, \& Thapar, 2012). Therefore, it is worthwhile to re-examine this. Second, a cognitive approach is one of the most tested models in understanding as well as in treating PTSD and depression. Cognitive factors such as perceived threat, maladaptive appraisal and cognitive coping (e.g., rumination, thought suppression) have been found to be robust in maintaining PTSD symptoms (Ehlers \& Clark, 2000; Lavi \& Solomon, 2005; Meiser-Stedman, Dalgleish, Smith, Yule, \& Glucksman, 2007; Spaccarelli, 1994; Stallard \& Smith, 2007). Likewise, negative cognitive style (e.g., rumination, selfblaming) is predictive of depression (Alloy et al., 2000; Alloy et al., 2006) whereas its effects on trauma-related depression symptoms are yet to be tested. Third, anxiety can lead to depression (Cole, Peeke, Martin, Truglio, \& Seroczynski, 1998); therefore, post-trauma anxiety levels were also included in our modeling. Last, it is prudent to have pre-trauma emotional wellbeing as the baseline in the model, in order to eliminate the chance of the observed PTSS and depression symptoms being the result of pre-existing mental health difficulties.

In summary, we hypothesized that PTSD and post-trauma depression are two partially independent reactions to trauma which share certain common mechanisms. To examine the elements of the relationship, the study used the trajectory modelling approach, where the differences and similarities were compared in three ways: 1) symptom changes in time (trajectories); 2) the synchronicity of the trajectories: and 3) their predictors.

\section{Method}

\section{Participants}

The study used longitudinal data collected by the Acute Stress Programme for Children and Teenagers (ASPECTS), a project set up to study acute PTSD among children and adolescents. Two previous studies have focused on the acute time frame at two week and two months (Meiser-Stedman et al., 2017, 2019); this study used the extended data collected at nine 
months. Participants aged eight to 17 years were recruited after attending an emergency department (ED) following a single event trauma including assault, road traffic accident (RTA) and accidental injury. Cases of chronic trauma exposure, intellectual disability, organic brain damage, significant self-harm and not being a fluent English speaker were excluded from the study. Participants who did not complete the questionnaires at two weeks were excluded from the present study.

The two key variables of the study were the severity of PTSD and depression symptoms after trauma. These were measured using the Child PTSD Symptom Scale (CPSS; Foa, Johnson, Feeny \& Treadwell, 2001) and the Short Mood and Feelings Questionnaire (SMFQ; Angold, Costello, Messer \& Pickles, 1995), respectively.

CPSS is a self-report questionnaire developed to assess PTSD symptoms for schoolage children. It is comprised of 24 items that can be divided into two parts. The first 17 items measure the type and frequency of PTSD symptoms (mapping directly on to DSM-IV criteria), while the other seven items measure the degree of impairment in functioning. It has shown high reliability and validity across various types of trauma (Foa et al., 2001; Gillihan, Aderka, Conklin, Capaldi, \& Foa, 2013; Nixon et al., 2013). A score of 16 was considered a clinical cutoff (Nixon et al., 2013). Of note, four items relating to dissociative symptoms (see appendix A) were added to the ASPECTS questionnaire to accommodate the then newly published DSM-5 PTSD dissociative subtype.

SMFQ is a short version (13 items) of the Mood and Feelings Questionnaire, an inventory that measures depressive symptoms in children and adolescents. Each item is rated on a 3-point scale: "true", "sometimes true", and "not true" with respect to their mood and feelings in the past two weeks. It has been proven to be an efficient discriminative tool for school-age children (Cheng, Cao, \& Su, 2009; Sharp, Goodyer, \& Croudace, 2006; Thabrew, 
Stasiak, Bavin, Frampton, \& Merry, 2018) through to late adolescents (Rhew et al., 2010; Turner, Joinson, Peters, Wiles, \& Lewis, 2014). A total score of eight or higher signifies clinical levels of depression (Angold et al., 1995).

Predictor measures were mostly derived from a few new measures developed in previous studies. All measure details can be found in Supplementary Appendix A: Novel Questionnaires Used in the Study. Pre-trauma emotional well-being (CPAS) was assessed based on 10 items from the Posttraumatic Adjustment Scale (O'Donnell et al., 2008) that indexes anxiety, low mood and anger. Peri-traumatic panic (CPP) was assessed using a 10item questionnaire addressing the symptoms associated with a panic disorder diagnosis (Meiser-Stedman et al., 2019). Post-trauma anxiety was assessed by Spence Children's Anxiety Scale (SCAS; Spence, 1998). Cognitive appraisal was assessed using Child Post-Traumatic Cognitions Inventory (CPTCI; Meiser-Stedman et al., 2009), a 25-item self-report designed to assess dysfunctional trauma-related cognitions. Thought suppression (Children's Thought Suppression Questionnaire, CTSQ) and rumination were assessed using five and three questionnaire items from a previous study that examined though control strategies and rumination in youth with acute stress disorder (Meiser-Stedman et al., 2014). Novel questionnaire items devised in a previous study (Meiser-Stedman et al., 2019) were used to assess adaptive processing and self-blaming. Adaptive processing, referring to deliberate efforts to mentally clarify what happened in the traumatic event, was assessed using a fiveitem measure (Children's Adaptive Processing Questionnaire, CAPQ; Cronbach's alpha = .73). Self-blame, referring to a cognitive process in which a person attributes the stress event to oneself, was assessed using a two-item measure (Cronbach's alpha $=.90$ ).

\section{Procedure}

The parents/caregivers of the children attending ED were initially contacted by letter 2-4 days post-ED attendance. Alongside the structured interview for acute stress disorder, 
consenting participants also completed self-report questionnaires at two weeks (T1), eight weeks (T2) and nine months (T3) via the telephone or online survey. The survey at T1 and T2 comprised the CPAS, CPTCI, TMQQ, SCAS, CPP, CPSS, CPS, SMFQ, CAPQ, CTSQ, rumination and self-blaming, whereas the nine months follow up only included the CPSS and SMFQ. Demographic information, nature of the incident, injury severity and medical treatment were obtained from the ED. Levels of CPSS and SMFQ at T1, T2 and T3 were used for trajectory modelling while values of predictive variables were all from T1.

\section{Statistical Analysis}

The data analysis followed several steps. First, the distribution of CPSS and SMFQ was checked in order to determine the distribution choice for trajectory modeling. We then used a GBTM program Proc Traj to run single modelling for CPSS and SMFQ separately to estimate their candidate models. These candidate models were assessed by their Bayesian Information Criterion (BIC) values along with other interpretive criteria so that we could choose a best fit model for each of the two measures. Next, a joint trajectory model was carried out based on the chosen individual models with a dropout option that compensated for missing data. The joint trajectory returned fine-tuned trajectory groups as final results, together with the conditional probability that indexed the connection between the CPSS and SMFQ trajectory groups. Finally, we utilized logistic regression analysis to investigate the link between the predictors and the high symptom groups. Details of each step are as follows.

Data Analysis Software - Proc Traj. Proc Traj is a SAS/STATA procedure developed by Jones, Nagin, and Roeder (2001). It uses a specialized application of finite mixture modeling to estimate trajectories and does not assume a one size-fits-all model for characterizing symptom onset and progression. Beside the basic modeling function, the package has been extended with functions such as dual-trajectory modeling (Jones \& Nagin, 2007). Detailed 
documentation of the Traj procedure can be found at https://www.andrew.cmu.edu/user/bjones/.

Distribution Estimation. CPSS and SMFQ scores are psychometric data with censored minimum and maximum values; they can also be treated as count data. Estimating the distribution of CPSS and SMFQ variables became necessary so that an appropriate modeling option (CNORM vs. ZIP) could be chosen. An R package, fitdistrplus (https://cran.rproject.org/web/packages/fitdistrplus/index.html) was employed to ascertain the distribution of the scores of first wave CPSS and SMFQ. Negative exponential distribution was considered the best fit for all six variables (see Appendix B), therefore ZIP distribution was chosen for the trajectory modeling.

Single Modeling. Given the exploratory nature of modeling, there is no guarantee a procedure will find a successful fit and so determining starting values becomes critical (Jones et al., 2001). Single modeling was used to approximate the parameters of the CPSS and SMFQ trajectories separately before embarking on the joint modeling. Based on previous findings in the literature, potential models with three and four groups were tested. A model of two groups was included as baseline for comparison.

For ZIP distribution, Proc Traj's statistical modeling assumes

$$
\ln \left(\lambda_{i t}^{j}\right)=\beta_{0}^{j}+\beta_{1}^{j} \text { Time }_{i t}+\beta_{2}^{j} \text { Time }_{i t}^{2}+\beta_{3}^{j} \text { Time }_{i t}^{3}+\beta_{4}^{j} \text { Time }_{i t}^{4}
$$

where $\lambda_{i t}^{j}$ is the event of interest $\mathrm{i}$ at time $\mathrm{t}$, given membership in group $\mathrm{j}$, and Time $_{i t}$ is the sampling time point at time $\mathrm{t}$ lapsed since the event. The model's coefficients$\beta_{0}^{j}, \beta_{1}^{j}, \beta_{2}^{j}, \beta_{3}^{j}$ and $\beta_{4}^{j}$ determine the shape of the trajectory. Since Proc Traj allows up to four degrees, our strategy was to probe the possible combinations of a group's polynomial order and to find their highest significant $(\mathrm{p}<.05)$ degree.

Model Selection. Once the single modeling was completed, a best-fit model was selected for each of the outcome measures. Although Jones et al. (2001) recommended the 
algorithm of using two times the change of the BIC values of the adjacent models as the criterion, we argued that it is equally important to realize that depending solely on a statistical figure might fail to capture a model that is clinically meaningful and succinct.

Joint Modeling. This was the last and the essential step of the modeling analysis. It was to refine the trajectories and to calculate the conditional probability based on Bayesian theorem in order to make immediate linkage between the trajectory groups of the PTSD and the depression. The configurations of the two selected models produced by the previous steps were fed into the joint modeling function. False convergence warning was given after the first run, hence a fine-tuning was needed. We used the option "detail" to obtain the parameters retuned from the first run, removed insignificant ones (whose $\mathrm{p}$ value $>.05$ ), and put the rest into a second run as starting values (see complete STATA script in Appendix C). The program adjusted well and the model was finalized.

Dropout Analysis. Attrition has been a challenge for longitudinal studies and data missingness requires careful handling or the results yielded will be at high risk of bias. Strategies for handling missing data may depend on whether the data are missing completely at random (MCAR), missing at random (MAR) or missing not at random (MNAR). In practice, the difference between MCAR, MAR and MNAR is often too elusive to ascertain (Graham, 2009). There was an attrition rate of approximately one third at nine months in our study and the randomness of the missing data was hard to estimate. Fortunately, Haviland, Jones, and Nagin (2011) extended the Proc Traj package with a dropout option. They demonstrated that nonrandom attrition, in which the dropout rate is uneven across the latent groups, has a consequential impact on modeling the group size. In an extreme case, a group might completely diminish. They further illustrated that the dropout extension was able to successfully optimize the model by taking account of differed dropout rates. Thus, we adopted the dropout option in 
modeling in the hope that it would not only estimate a better model but also offer informative judgment on the missing data.

Predictive Factor Analysis. After the joint modeling, each case was categorized into one PTSD group and one depression group according to the course of their symptoms over the nine months, where their membership profiles were labeled as low (1), medium (2) or high $(3)^{1}$. A multinomial logistic regression analysis was then deployed to calculate the relative risk ratio of falling into the high symptom group compared to the low symptom group according to various early signs. Specifically, age and gender, measures of pre-trauma emotional wellbeing (CPAS), peri-trauma panic (CPP), post-trauma anxiety (SCAS), appraisal (CPTCI), rumination, adaptive-processing and self-blaming were used as predictors.

Trauma severity, a common factor that may influence the post-trauma response, was not included in the modelling. The previous study using the same sample revealed that objective indices of trauma severity (number of injuries, sustaining a fracture, being seen in 'resus', sustaining an injury with permanent loss of function) were not significantly related to PTSD symptoms (Meiser-Stedman et al., 2019). That study suggested that the cognitive processes (peritraumatic panic, post-traumatic rumination, negative appraisals and adaptive thinking) played an important role in the onset and maintenance of PTSS; thus, the present study focused on examining the impact of the cognitive elements. Regression analysis also confirmed that trauma severity was not associated with depression (SMFQ) scores; model outputs are listed in Table S3.

It is worth noting that the focus of this study lies in the relative difference in the types and the magnitude of the predictors modelled as opposed to their individual correlation, 
because variables interplay and the correlation would be meaningless without controlling covariation. For example, PTSD was once under the umbrella of anxiety disorders, and SCAS captures overlapping symptoms such as avoidance, arousal and panic, so it would be no coincidence if anxiety is a significant independent correlation, but its predictive power would be in question.

\section{Results}

\section{Descriptive statistics}

The data analysis included 217 cases, of which 124 were males and 93 were females. Participants were aged between 8.01 and 17.97 years $(M=14.09, \mathrm{SD}=2.96)$. The traumatic events involved 98 cases of RTA, 71 cases of accidental injuries, 35 cases of assault, 11 cases of dog attacks and 2 cases of acute medical emergency. At T2, there were 13 cases missing CPSS scores and 14 cases missing SMFQ scores. At T3, 58 cases missed both CPSS and SMFQ scores.

\section{Model Selection}

\section{Insert Figure 1}

As illustrated in Table S1, for the CPSS trajectories, the BIC criterion favored models comprising four groups; for the SMFQ there was no significant difference (i.e., the BIC difference was less than 10). Nevertheless, we believed the three-group model was optimal for several reasons. Figure 1 presents the two proposed models: three groups vs four groups (see Figure 1a for CPSS and Figure 1b for SMFQ). The two models were similar in some key regards - they both encompassed a consistently low score group and high score group with broadly equivalent group size (29.3\% vs. $26.1 \%$ for the low score group and $21.1 \%$ vs. $17.1 \%$ for the high score group in PTSD; $19.6 \%$ vs. $17.6 \%$ for the high score group in depression). Except for the high score groups, the other groups all demonstrated a trend of reaching scores within a narrow range at nine months regardless of their starting points. The main difference 
between the three- and four-group models concerned the medium groups. The three-group options recommended one medium group whereas two separate groups were proposed by the four-group options. Although they appear to start at various points at T1, the nuance does not seem to bear clinical significance given the clinical cutoff is 16 for CPSS and eight for SMFQ, and both medium groups in the four-group models started below these cut-offs. The four-group options also had larger CIs (areas between the dashed lines). Thus, we favored the more succinct three-group model. Moreover, the more parsimonious models would be beneficial when it came to joint modelling, because when linking the outcomes of the two trajectories, the proliferation of probability matrices could easily become unmanageable. Specifically, the four PTSD and four depression groups produce 16 combinations while the three-group option only produces nine. Those seven extra combinations are the hybrids from the medium groups, which can be expected to be almost identical.

\section{CPSS \& SMFQ Joint Modeling}

As mentioned earlier, the shape of the trajectory of each group is determined by a vector of coefficients $\left(\beta_{0}^{j}, \beta_{1}^{j}, \beta_{2}^{j}, \beta_{3}^{j}\right.$ and $\left.\beta_{4}^{j}\right)$. Our model attained significant $(\mathrm{p}<.00001)$ coefficients for all trajectory groups and their drop out polynomials (see complete output in Supplementary Table S1). Conditional as well as joint membership probability were also reported. Nontechnical interpretations are described as follows.

\section{PTSD Trajectory}

\section{Insert Figure 2}

\section{Insert Table 1}

The final joint model yielded three distinct PTSD trajectory groups including a large group (42.4\% of the sample size) with persistently low CPSS scores, a medium group (35.6\%) with marginally significant CPSS score at week two which dropped below the clinical cutoff at week eight, and a high group taking up $21.9 \%$ of the participants presenting marked distress 
at week two and week eight. At nine months, the scores of the three groups were in the nonclinical range.

\section{Depression Trajectory}

Similarly, the joint model produced three depression trajectory groups (low, medium and high) comprising $45.9 \%, 34.0 \%$ and $20.1 \%$ of the participants, respectively. In contrast to the low and medium groups, whose depression level remained persistently low, the SMFQ score of the high depression group at nine months ( $\mathrm{M}=7.96,95 \% \mathrm{CI}[7.32,11.17])$ was still at the clinical cutoff.

\section{Conditional Group Membership}

In probability theory, conditional probability is a measure of the probability of an event occurring given that another event has occurred. If we knew a case was categorized as low PTSS, the probability of its belonging to the low, medium and high depression symptom groups would be $81.2 \%, 29.6 \%$ and $5.4 \%$, respectively. Conversely, the probability of its belonging to the PTSD group conditional on a low depression group would be $74.6 \%, 21.7 \%$ and $3.3 \%$, respectively. Figure 4 lists the conditional probabilities of all the possible combinations. In addition, the model also reported the joint probability of belonging to the PTSS group and the depression group (Table 4).

\section{Dropout Model}

A flat lineal model was proposed by the dropout analysis suggesting an equal rate of attrition at wave two and three. Estimated dropout rates of the PTSD groups were 14.1\%, $15.5 \%, 30.2 \%$, and those of the depression groups were $16.3 \%, 11.9 \%$ and $65.1 \%$, respectively.

\section{Predictive Factors}

Using low symptom groups as the referent, gender, age, CPAS, CPP, SCAS, CPTCI, rumination, thought suppression, adaptive processing and self-blaming items were entered as independent variables into two multinomial logistic regressions (see STATA scripts in 
Appendix D) to predict the PTSD and depression trajectory outcome, in particular for high symptom groups. The relative risk ratio in the model estimates for one unit increase in each of the predictive factors the change in the probability of falling into the high symptom group rather than the low symptom group, given that the other variables in the model are held constant.

The statistically significant predictors $(\mathrm{p}<.05)$, ordered from strongest to weakest, for the high PTSD group were: peri-event panic $(\mathrm{RR}=2.09$, 95\% CI $[1.38,3.19])$, rumination $(\mathrm{RR}=1.60,95 \% \mathrm{CI}[1.076189,2.394697])$, thought suppression $(\mathrm{RR}=1.27,95 \% \mathrm{CI}[1.04$, 1.56]) and appraisal ( $\mathrm{RR}=1.26,95 \% \mathrm{CI}[1.12,1.42])$. The statistically significant predictors for the high depression group (also from strongest to weakest) were: appraisal ( $\mathrm{RR}=1.31,95 \% \mathrm{CI}$ $[1.15,1.48])$, pre-trauma emotional wellbeing $(\mathrm{RR}=1.24,95 \% \mathrm{CI}[1.03,1.49])$, and posttrauma anxiety $(\mathrm{RR}=1.20,95 \% \mathrm{CI}[1.09,1.34])$. Gender and age did not predict PTSS or depression (Table 2).

\section{Discussion}

The study investigated the natural recovery trajectories of PTSS and depression symptoms for the nine months period following a single event trauma. Overall, our model suggested that all participants' PTSD symptoms reduced to non-clinical level, whereas $20 \%$ of them presented with enduring depression. The PTSS trajectories finding was consonant with the prototypical trauma response pattern lodged since 2004 (Bonanno, 2004), although no delayed onset cluster was detected in our sample. The majority (80\%) were observed to be consistently displaying low symptoms or to be able to recover within two months, while about one fifth of participants experienced high level PTSD symptoms but managed to reach the recovery range within nine months. Unlike the other two youth studies (de Roon-Cassini et al., 2010; Punamäki et al., 2015), there was no chronic/increase group, which might be explained by the relatively mild trauma and the absence of ongoing stress or loss in our sample. Another 
reason could be that participants with elevated PTSD symptoms were referred to intervention and were excluded from the study.

The depression trajectories were quite different. The three trajectories all described a steady decline but the divergence between the high depression group (20\%) and the rest was such that the high depression group was chronically depressed for nine months, during which time the other two groups demonstrated only mild symptoms. Such a dichotomous pattern has not been apparent in previous trajectory studies.

In respect of the relationship between the PTSS and depression trajectories, the conditional membership analysis reported high synchronicity: low PTSS participants were highly likely to be classified in the low depression group, while a participant who experienced high PTSS was anticipated to be in the highly depressed group. Similarly, being in the high depression group predicted being in the more severe PTSD symptoms group.

Intriguingly, the high PTSS and depression groups shared few predictive factors. Rumination in general is considered a transdiagnostic feature associated with depression and PTSD, and it was strongly related to PTSD in this study although it did not predict depression in our model. This phenomenon suggests that a certain subtype(s) of rumination may maintain PTSD but not depression. Birrer and Michael (2011) conducted a study examining the characteristics of rumination such as duration and content in PTSD and depression, and found that rumination served as a powerful internal trigger for intrusive memories in PTSD, but not in depression. Constructions of various types of rumination (e.g. depressive rumination, stressreactive rumination) have been suggested and their clinical impact needs further investigation.

In line with the literature, peri-trauma panic (perceived threat), thought suppression and negative appraisal were linked with high PTSS, while only negative appraisals was a factor that was associated with both high symptom groups. This finding confirms that cognitive appraisal plays a role in maintaining broader posttrauma psychopathology (Hiller et al., 2019). 
Hamilton et al. (2012) integrated findings from a large body of neuroimaging research and proposed that depression is sustained by the increased salience of negative information leading to biased appraisal. Combined with the heightened sense of threat (e.g., intrusive memory, hypervigilance) in PTSD, which serves as an ongoing source of negative information, appraisal may be central to understanding PTSD-depression comorbidity.

Apart from negative appraisal, the other two predictors of depression were pre-trauma emotional difficulties and post-trauma anxiety, which were unexpectedly not correlated with PTSS when controlling for appraisals. This result suggested that PTSD is distinct from other anxiety disorders (e.g., panic disorder, social anxiety, GAD) not only at the symptom level but also in the vulnerabilities and maintenance mechanisms. This is consistent with neuroimaging evidence that PTSD is less explained by a mere fear response in the amygdala and insula, and features prominent hypoactivations in the regions associated with the experience or regulation of emotion (Etkin \& Wager, 2007).

In summarizing the PTSS-depression relationship observed in the study, we concluded that PTSS and depression are two distinct, but overlapping, responses to a traumatic stressor, and that they are maintained by different processes. This conclusion is based on 1) the high synchronicity in their trajectories, and 2) few mutual predictors. Negative appraisals appeared to play a critical role bridging their mechanisms. Anxiety manifested as the second strongest predictor of depression though we speculated that it could be a mere byproduct of the overlapping presentations of physiological arousal and avoidance in both anxiety disorders and PTSD.

Lastly, our study is the first to have looked at trajectory and attrition rate in the field and found that the more severe symptoms a participant has, the more likely they will drop out from the study. We conjecture that this rule may hold universally in longitudinal research and clinical trials, and the consequences can be serious. The immediate consequence is that without 
correction the averages of the examined measures will be lower than their real means, and other prime parameters of the sampled distribution, such as standard deviation, will be altered, making inferred statistical interpretation less accurate. Therefore, this conclusion supports handling missing data with great caution and, if possible, applying appropriate statistical methods (e.g. dropout modeling or imputation) to minimize the impact.

\section{Clinical Implication}

In the case of acute posttraumatic stress, depression can be a more alarming condition than PTSD symptoms, as PTSS tends to diminish given time, whereas young trauma victims may endure persisting depression if left untreated. Counter-intuitively, an anxious/withdrawn child is not more vulnerable to PTSD, but is instead more vulnerable to depression. The large dropout rate in the high depression group (60\%) emphasizes the importance of catching early signs of depression, because withdrawal from clinical research may suggest they are also prone to dropout from treatment or are more reluctant to seek professional help.

Most importantly, negative appraisal being the only predictor for high PTSS as well as high depression suggests a possible effective treatment, addressing PTSS and depression holistically by tackling the appraisal component.

\section{Limitations}

The study had several discernible limitations. First of all, the data were limited to children and adolescents mainly following a one-off, non-interpersonal trauma. Thus, the interpretation of the results may not apply to interpersonal or multiple traumas. Second, for ethical reasons, the dataset was only able to track the natural course of participants with relatively mild symptoms. Ten cases with high PTSD measurement scores were referred for treatment and were therefore excluded from the study. The trajectories that emerged in this study may, therefore, not represent the clinical population. Third, the CPSS and SMFQ measures were sampled at two weeks, two months and nine months, missing the six months 
watershed at which recovery and non-recovery divide in young populations (Hiller et al., 2016). Moreover, the drop out modeling suggested the equal dropout rate at T2 and T3, which did not fit the actual data perfectly (the missing rate at T2 was 13/14 cases and 58 cases at T3). Consequently, the estimated means at $\mathrm{T} 2$ could be higher than their true values as the joint modeling compensates for the missing data by applying the high score, high dropout formulation.

\section{Conclusion}

Within children and adolescents exposed to single event trauma resulting in minor physical injury, the majority were able to recover without intervention, although about one fifth presented with symptoms of lasting depression at nine months follow up. By examining predictors of high symptom groups, negative cognitive appraisal appeared to be a shared risk factor linking PTSS and depression. Anxiety did not predict PTSS but did predict depression. 
Paragraph: use this for the first paragraph in a section, or to continue after an extract.

New paragraph: use this style when you need to begin a new paragraph.

Display quotations of over 40 words, or as needed.

- For bulleted lists

(1) For numbered lists

Displayed equation

\section{Heading 2: use this style for level two headings}

Heading 3: use this style for level three headings

Heading 4: create the heading in italics. Run the text on after a punctuation mark.

Acknowledgements, avoiding identifying any of the authors prior to peer review

1. This is a note. The style name is Footnotes, but it can also be applied to endnotes.

References: see the journal's instructions for authors for details on style 
Table 1. Type your title here.

Figure 1. Type your title here. Obtain permission and include the acknowledgement required by the copyright holder if a figure is being reproduced from another source. 


\section{References}

Abela, J. R., Aydin, C. M., \& Auerbach, R. P. (2007). Responses to depression in children: Reconceptualizing the relation among response styles. Journal of abnormal child psychology, 35(6), 913-927.

Allgood-Merten, B., Lewinsohn, P. M., \& Hops, H. (1990). Sex differences and adolescent depression. Journal of abnormal psychology, 99(1), 55.

Alloy, L. B., Abramson, L. Y., Hogan, M. E., Whitehouse, W. G., Rose, D. T., Robinson, M. S., Lapkin, J. B. (2000). The Temple-Wisconsin Cognitive Vulnerability to Depression Project: Lifetime history of Axis I psychopathology in individuals at high and low cognitive risk for depression. Journal of abnormal psychology, 109(3), 403.

Alloy, L. B., Abramson, L. Y., Whitehouse, W. G., Hogan, M. E., Panzarella, C., \& Rose, D. T. (2006). Prospective incidence of first onsets and recurrences of depression in individuals at high and low cognitive risk for depression. Journal of abnormal psychology, 115(1), 145.

Angold, A., Costello, E. J., Messer, S. C., \& Pickles, A. (1995). Development of a short questionnaire for use in epidemiological studies of depression in children and adolescents. International journal of methods in psychiatric research.

Betancourt, T. S., McBain, R. K., \& Brennan, R. T. (2014). Trajectories of externalizing problems among war-affected youth in Sierra Leone: results from a longitudinal study. Aggression and Violent Behavior, 19(6), 708-714.

Betancourt, T. S., McBain, R., Newnham, E. A., \& Brennan, R. T. (2013). Trajectories of internalizing problems in war - affected Sierra Leonean youth: Examining conflict and postconflict factors. Child development, 84(2), 455-470. 
Birrer, E., \& Michael, T. (2011). Rumination in PTSD as well as in traumatized and nontraumatized depressed patients: A cross-sectional clinical study. Behavioural and Cognitive Psychotherapy, 39(4), 381-397.

Bleich, A., Koslowsky, M., Dolev, A., \& Lerer, B. (1997). Post-traumatic stress disorder and depression: An analysis of comorbidity. The British Journal of Psychiatry, 170(5), 479-482.

Bombardier, C. H., Hoekstra, T., Dikmen, S., \& Fann, J. R. (2016). Depression trajectories during the first year after traumatic brain injury. Journal of neurotrauma, 33(23), 2115-2124.

Bonanno, G. A. (2004). Loss, trauma, and human resilience: have we underestimated the human capacity to thrive after extremely aversive events? American psychologist, 59(1), 20.

Bonanno, G. A., Kennedy, P., Galatzer-Levy, I. R., Lude, P., \& Elfström, M. L. (2012). Trajectories of resilience, depression, and anxiety following spinal cord injury. Rehabilitation psychology, 57(3), 236.

Bonanno, G. A., \& Mancini, A. D. (2012). Beyond resilience and PTSD: Mapping the heterogeneity of responses to potential trauma. Psychological Trauma: Theory, Research, Practice, and Policy, 4(1), 74.

Breslau, N., Davis, G. C., Andreski, P., Peterson, E. L., \& Schultz, L. R. (1997). Sex differences in posttraumatic stress disorder. Archives of general psychiatry, 54(11), 1044-1048.

Breslau, N., Davis, G. C., Peterson, E. L., \& Schultz, L. R. (2000). A second look at comorbidity in victims of trauma: The posttraumatic stress disorder-major depression connection. Biological psychiatry, 48(9), 902-909. 
Cheng, P.-X., Cao, F.-L., \& Su, L.-Y. (2009). Reliability and validity of the Short Mood and Feelings Questionnaire in Chinese adolescents. Chinese Mental Health Journal.

Clark, D. B., \& Kirisci, L. (1996). Posttraumatic stress disorder, depression, alcohol use disorders and quality of life in adolescents. Anxiety, 2(5), 226-233.

Clark, D. B., Pollock, N., Bukstein, O. G., Mezzich, A. C., Bromberger, J. T., \& Donovan, J. E. (1997). Gender and comorbid psychopathology in adolescents with alcohol dependence. Journal of the American Academy of Child \& Adolescent Psychiatry, 36(9), 1195-1203.

Cole, D. A., Peeke, L. G., Martin, J. M., Truglio, R., \& Seroczynski, A. (1998). A longitudinal look at the relation between depression and anxiety in children and adolescents. Journal of consulting and clinical psychology, 66(3), 451.

Contractor, A. A., Layne, C. M., Steinberg, A. M., Ostrowski, S. A., Ford, J. D., \& Elhai, J. D. (2013). Do gender and age moderate the symptom structure of PTSD? Findings from a national clinical sample of children and adolescents. Psychiatry Research, $210(3), 1056-1064$.

Cook, A., Spinazzola, J., Ford, J., Lanktree, C., Blaustein, M., Cloitre, M., . . Liautaud, J. (2017). Complex trauma in children and adolescents. Psychiatric annals, 35(5), 390398.

Cummings, C. M., Caporino, N. E., \& Kendall, P. C. (2014). Comorbidity of anxiety and depression in children and adolescents: 20 years after. Psychological bulletin, 140(3), 816.

deRoon-Cassini, T. A., Mancini, A. D., Rusch, M. D., \& Bonanno, G. A. (2010). Psychopathology and resilience following traumatic injury: a latent growth mixture model analysis. Rehabilitation psychology, 55(1), 1. 
Ehlers, A., \& Clark, D. M. (2000). A cognitive model of posttraumatic stress disorder. Behaviour research and therapy, 38(4), 319-345.

Etkin, A., \& Wager, T. D. (2007). Functional neuroimaging of anxiety: a meta-analysis of emotional processing in PTSD, social anxiety disorder, and specific phobia. American Journal of psychiatry, 164(10), 1476-1488.

Fan, F., Long, K., Zhou, Y., Zheng, Y., \& Liu, X. (2015). Longitudinal trajectories of posttraumatic stress disorder symptoms among adolescents after the Wenchuan earthquake in China. Psychological medicine, 45(13), 2885.

Flory, J. D., \& Yehuda, R. (2015). Comorbidity between post-traumatic stress disorder and major depressive disorder: alternative explanations and treatment considerations. Dialogues in clinical neuroscience, 17(2), 141.

Foa, E. B., Johnson, K. M., Feeny, N. C., \& Treadwell, K. R. (2001). The Child PTSD Symptom Scale: A preliminary examination of its psychometric properties. Journal of clinical child psychology, 30(3), 376-384.

Galatzer-Levy, I. R., Huang, S. H., \& Bonanno, G. A. (2018). Trajectories of resilience and dysfunction following potential trauma: A review and statistical evaluation. Clinical Psychology Review, 63, 41-55

Gillihan, S. J., Aderka, I. M., Conklin, P. H., Capaldi, S., \& Foa, E. B. (2013). The Child PTSD Symptom Scale: psychometric properties in female adolescent sexual assault survivors. Psychological assessment, 25(1), 23.

Graham, J. W. (2009). Missing data analysis: Making it work in the real world. Annual review of psychology, 60, 549-576.

Green, B. L., Korol, M., Grace, M. C., Vary, M. G., Leonard, A. C., Gleser, G. C., \& Smitson-Cohen, S. (1991). Children and disaster: Age, gender, and parental effects on 
PTSD symptoms. Journal of the American Academy of Child \& Adolescent Psychiatry, 30(6), 945-951.

Greene, T., Gelkopf, M., Grinapol, S., Werbeloff, N., Carlson, E., \& Lapid, L. (2017). Trajectories of traumatic stress symptoms during conflict: A latent class growth analysis. Journal of affective disorders, 220, 24-30.

Hamilton, J. P., Etkin, A., Furman, D. J., Lemus, M. G., Johnson, R. F., \& Gotlib, I. H. (2012). Functional neuroimaging of major depressive disorder: a meta-analysis and new integration of baseline activation and neural response data. American Journal of psychiatry, 169(7), 693-703.

Haviland, A. M., Jones, B. L., \& Nagin, D. S. (2011). Group-based trajectory modeling extended to account for nonrandom participant attrition. Sociological methods \& research, 40(2), 367-390.

Hiller, R. M., Creswell, C., Meiser-Stedman, R., Lobo, S., Cowdrey, F., Lyttle, M. D., . . Halligan, S. L. (2019). A longitudinal examination of the relationship between trauma-related cognitive factors and internalising and externalising psychopathology in physically injured children. Journal of abnormal child psychology, 47(4), 683-693.

Hiller, R. M., Meiser-Stedman, R., Fearon, P., Lobo, S., McKinnon, A., Fraser, A., \& Halligan, S. L. (2016). Research Review: Changes in the prevalence and symptom severity of child post-traumatic stress disorder in the year following trauma-a metaanalytic study. Journal of Child Psychology and Psychiatry, 57(8), 884-898.

Hong, S. B., Youssef, G. J., Song, S. H., Choi, N. H., Ryu, J., McDermott, B., ... \& Yoo, H. J. (2014). Different clinical courses of children exposed to a single incident of psychological trauma: A 30 - month prospective follow - up study. Journal of Child Psychology and Psychiatry, 55(11), 1226-1233. 
Jones, B. L., \& Nagin, D. S. (2007). Advances in group-based trajectory modeling and an SAS procedure for estimating them. Sociological methods \& research, 35(4), 542571.

Jones, B. L., Nagin, D. S., \& Roeder, K. (2001). A SAS procedure based on mixture models for estimating developmental trajectories. Sociological methods \& research, 29(3), 374-393.

Jovanovic, T., Norrholm, S. D., Blanding, N. Q., Davis, M., Duncan, E., Bradley, B., \& Ressler, K. J. (2010). Impaired fear inhibition is a biomarker of PTSD but not depression. Depression and anxiety, 27(3), 244-251.

Kearns, M. C., Ressler, K. J., Zatzick, D., \& Rothbaum, B. O. (2012). Early interventions for PTSD: a review. Depression and anxiety, 29(10), 833-842.

La Greca, A. M., Lai, B. S., Llabre, M. M., Silverman, W. K., Vernberg, E. M., \& Prinstein, M. J. (2013, August). Children's postdisaster trajectories of PTS symptoms: Predicting chronic distress. In Child \& youth care forum (Vol. 42, No. 4, pp. 351369). Springer US.

Lai, B. S., La Greca, A. M., Auslander, B. A., \& Short, M. B. (2013). Children's symptoms of posttraumatic stress and depression after a natural disaster: Comorbidity and risk factors. Journal of affective disorders, 146(1), 71-78.

Lavi, T., \& Solomon, Z. (2005). Palestinian youth of the Intifada: PTSD and future orientation. Journal of the American Academy of Child \& Adolescent Psychiatry, 44(11), 1176-1183.

Lauterbach, D., \& Armour, C. (2016). Symptom trajectories among child survivors of maltreatment: Findings from the Longitudinal Studies of Child Abuse and Neglect (LONGSCAN). Journal of abnormal child psychology, 44(2), 369-379. 
Le Brocque, R. M., Hendrikz, J., \& Kenardy, J. A. (2009). The course of posttraumatic stress in children: Examination of recovery trajectories following traumatic injury. Journal of Pediatric Psychology, 35(6), 637-645.

Litz, B. T., Gray, M. J., Bryant, R. A., \& Adler, A. B. (2002). Early intervention for trauma: Current status and future directions. Clinical psychology: science and practice, 9(2), 112-134.

Maikovich, A. K., Koenen, K. C., \& Jaffee, S. R. (2009). Posttraumatic stress symptoms and trajectories in child sexual abuse victims: An analysis of sex differences using the National Survey of Child and Adolescent Well-Being. Journal of abnormal child psychology, 37(5), 727-737.

Mazza, J. J., \& Reynolds, W. M. (1999). Exposure to violence in young inner-city adolescents: Relationships with suicidal ideation, depression, and PTSD symptomatology. Journal of abnormal child psychology, 27(3), 203-213.

Meiser-Stedman, R., Dalgleish, T., Smith, P., Yule, W., \& Glucksman, E. (2007). Diagnostic, demographic, memory quality, and cognitive variables associated with acute stress disorder in children and adolescents. Journal of abnormal psychology, 116(1), 65.

Meiser-Stedman, R., McKinnon, A., Dixon, C., Boyle, A., Smith, P., \& Dalgleish, T. (2017). Acute stress disorder and the transition to posttraumatic stress disorder in children and adolescents: Prevalence, course, prognosis, diagnostic suitability, and risk markers. Depression and anxiety, 34(4), 348-355.

Meiser-Stedman, R., McKinnon, A., Dixon, C., Boyle, A., Smith, P., \& Dalgleish, T. (2019). A core role for cognitive processes in the acute onset and maintenance of posttraumatic stress in children and adolescents. Journal of Child Psychology and Psychiatry. 
Meiser-Stedman, R., Smith, P., Bryant, R., Salmon, K., Yule, W., Dalgleish, T., \& Nixon, R. D. (2009). Development and validation of the child post-traumatic cognitions inventory (CPTCI). Journal of Child Psychology and Psychiatry, 50(4), 432-440.

Nagin, D. S. (1999). Analyzing developmental trajectories: a semiparametric, group-based approach. Psychological methods, 4(2), 139.

Nagin, D. S., \& Odgers, C. L. (2010). Group-based trajectory modeling in clinical research. Annual review of clinical psychology, 6.

Nixon, R. D., Meiser-Stedman, R., Dalgleish, T., Yule, W., Clark, D. M., Perrin, S., \& Smith, P. (2013). The Child PTSD Symptom Scale: An update and replication of its psychometric properties. Psychological assessment, 25(3), 1025.

Norris, F. H., Tracy, M., \& Galea, S. (2009). Looking for resilience: Understanding the longitudinal trajectories of responses to stress. Social science \& medicine, 68(12), 2190-2198.

Nugent, N. R., Saunders, B. E., Williams, L. M., Hanson, R., Smith, D. W., \& Fitzgerald, M. M. (2009). Posttraumatic stress symptom trajectories in children living in families reported for family violence. Journal of traumatic stress, 22(5), 460-466.

O'Donnell, M. L., Creamer, M. C., Parslow, R., Elliott, P., Holmes, A. C., Ellen, S., . . Bryant, R. A. (2008). A predictive screening index for posttraumatic stress disorder and depression following traumatic injury. Journal of consulting and clinical psychology, 76(6), 923.

Osofsky, J. D., Osofsky, H. J., Weems, C. F., King, L. S., \& Hansel, T. C. (2015). Trajectories of post-traumatic stress disorder symptoms among youth exposed to both natural and technological disasters. Journal of child psychology and psychiatry, 56(12), 1347-1355. 
Punamäki, R.-L., Palosaari, E., Diab, M., Peltonen, K., \& Qouta, S. R. (2015). Trajectories of posttraumatic stress symptoms (PTSS) after major war among Palestinian children: Trauma, family-and child-related predictors. Journal of affective disorders, 172, 133140.

Rhew, I. C., Simpson, K., Tracy, M., Lymp, J., McCauley, E., Tsuang, D., \& Vander Stoep, A. (2010). Criterion validity of the Short Mood and Feelings Questionnaire and oneand two-item depression screens in young adolescents. Child and adolescent psychiatry and mental health, 4(1), 8 .

Rytwinski, N. K., Scur, M. D., Feeny, N. C., \& Youngstrom, E. A. (2013). The co-occurrence of major depressive disorder among individuals with posttraumatic stress disorder: A meta-analysis. Journal of traumatic stress, 26(3), 299-309.

Saluja, G., Iachan, R., Scheidt, P. C., Overpeck, M. D., Sun, W., \& Giedd, J. N. (2004). Prevalence of and risk factors for depressive symptoms among young adolescents. Archives of pediatrics \& adolescent medicine, 158(8), 760-765.

Santiago, P. N., Ursano, R. J., Gray, C. L., Pynoos, R. S., Spiegel, D., Lewis-Fernandez, R., ... \& Fullerton, C. S. (2013). A systematic review of PTSD prevalence and trajectories in DSM-5 defined trauma exposed populations: intentional and non-intentional traumatic events. PloS one, 8(4), e59236.

Self-Brown, S., Lai, B. S., Thompson, J. E., McGill, T., \& Kelley, M. L. (2013). Posttraumatic stress disorder symptom trajectories in Hurricane Katrina affected youth. Journal of affective disorders, 147(1-3), 198-204.

Shalev, A. Y., Freedman, S., Peri, T., Brandes, D., Sahar, T., Orr, S. P., \& Pitman, R. K. (1998). Prospective study of posttraumatic stress disorder and depression following trauma. American Journal of psychiatry, 155(5), 630-637. 
Sharp, C., Goodyer, I. M., \& Croudace, T. J. (2006). The Short Mood and Feelings Questionnaire (SMFQ): a unidimensional item response theory and categorical data factor analysis of self-report ratings from a community sample of 7-through 11-yearold children. Journal of abnormal child psychology, 34(3), 365-377.

Spaccarelli, S. (1994). Stress, appraisal, and coping in child sexual abuse: A theoretical and empirical review. Psychological bulletin, 116(2), 340.

Spence, S. H. (1998). A measure of anxiety symptoms among children. Behaviour research and therapy, 36(5), 545-566.

Spinhoven, P., Penninx, B. W., van Hemert, A. M., de Rooij, M., \& Elzinga, B. M. (2014). Comorbidity of PTSD in anxiety and depressive disorders: Prevalence and shared risk factors. Child abuse \& neglect, 38(8), 1320-1330.

Stallard, P., \& Smith, E. (2007). Appraisals and cognitive coping styles associated with chronic post-traumatic symptoms in child road traffic accident survivors. Journal of Child Psychology and Psychiatry, 48(2), 194-201.

Stallard, P., Velleman, R., Salter, E., Howse, I., Yule, W., \& Taylor, G. (2006). A randomised controlled trial to determine the effectiveness of an early psychological intervention with children involved in road traffic accidents. Journal of Child Psychology and Psychiatry, 47(2), 127-134.

Stander, V. A., Thomsen, C. J., \& Highfill-McRoy, R. M. (2014). Etiology of depression comorbidity in combat-related PTSD: a review of the literature. Clinical psychology review, 34(2), 87-98.

Thabet, A. A. M., Abed, Y., \& Vostanis, P. (2004). Comorbidity of PTSD and depression among refugee children during war conflict. Journal of Child Psychology and Psychiatry, 45(3), 533-542. 
Thabrew, H., Stasiak, K., Bavin, L. M., Frampton, C., \& Merry, S. (2018). Validation of the Mood and Feelings Questionnaire (MFQ) and Short Mood and Feelings Questionnaire (SMFQ) in New Zealand help-seeking adolescents. International journal of methods in psychiatric research, e1610.

Thapar, A., Collishaw, S., Pine, D. S., \& Thapar, A. K. (2012). Depression in adolescence. The Lancet, 379(9820), 1056-1067.

Trickey, D., Siddaway, A. P., Meiser-Stedman, R., Serpell, L., \& Field, A. P. (2012). A metaanalysis of risk factors for post-traumatic stress disorder in children and adolescents. Clinical psychology review, 32(2), 122-138.

Turner, N., Joinson, C., Peters, T. J., Wiles, N., \& Lewis, G. (2014). Validity of the Short Mood and Feelings Questionnaire in late adolescence. Psychological assessment, $26(3), 752$.

Twisk, J., \& Hoekstra, T. (2012). Classifying developmental trajectories over time should be done with great caution: a comparison between methods. Journal of clinical epidemiology, 65(10), 1078-1087.

Vibhakar, V., Allen, L. R., Gee, B., \& Meiser-Stedman, R. (2019). A systematic review and meta-analysis on the prevalence of depression in children and adolescents after exposure to trauma. Journal of affective disorders, 255, 77-89. doi: 10.1016/j.jad.2019.05.005

Wang, C. W., Chan, C. L., \& Ho, R. T. (2013). Prevalence and trajectory of psychopathology among child and adolescent survivors of disasters: a systematic review of epidemiological studies across 1987-2011. Social psychiatry and psychiatric epidemiology, 48(11), 1697-1720. 
Trajectories of Post-traumatic Stress and Depression Following Accident: Course and Risk Factors
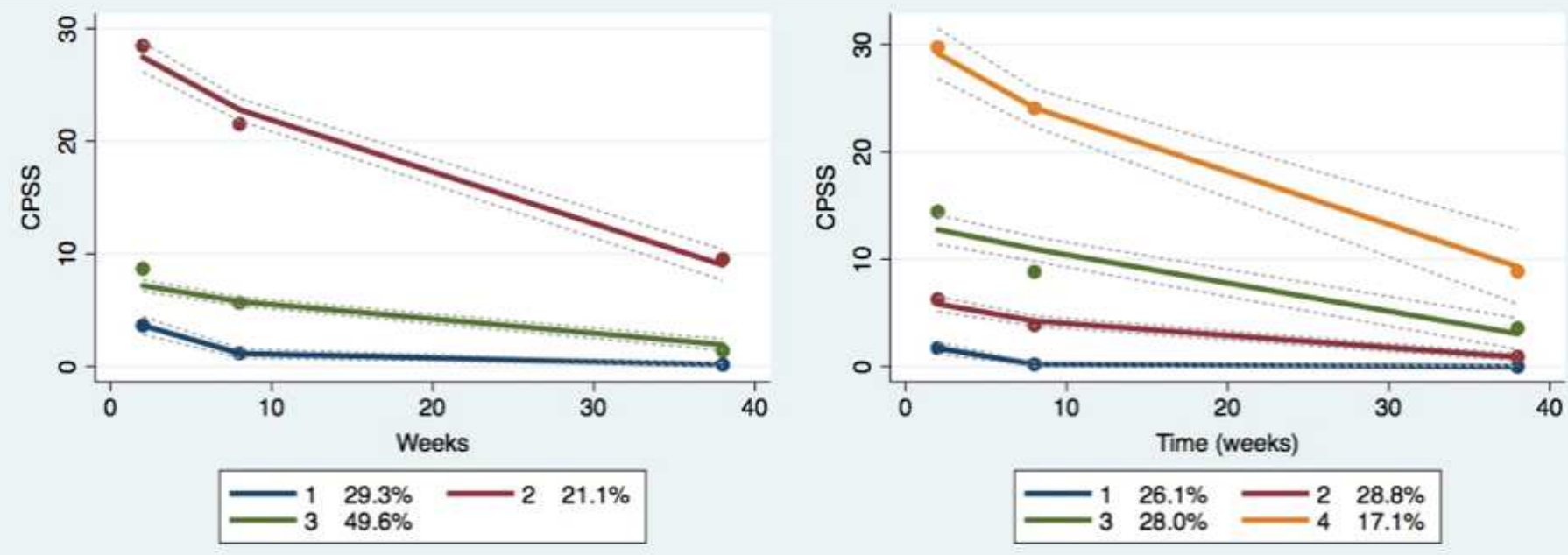

Figure 1a. Candidate models of SPSS
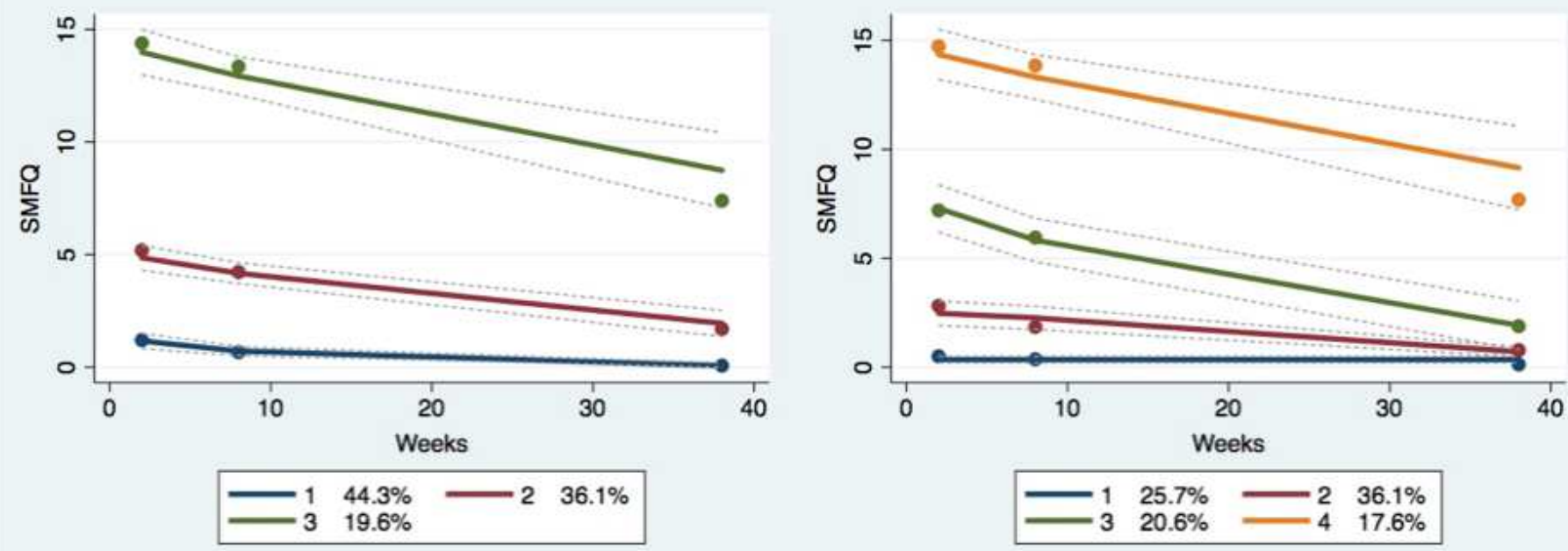

Figure 1b. Candidate models of SMFQ
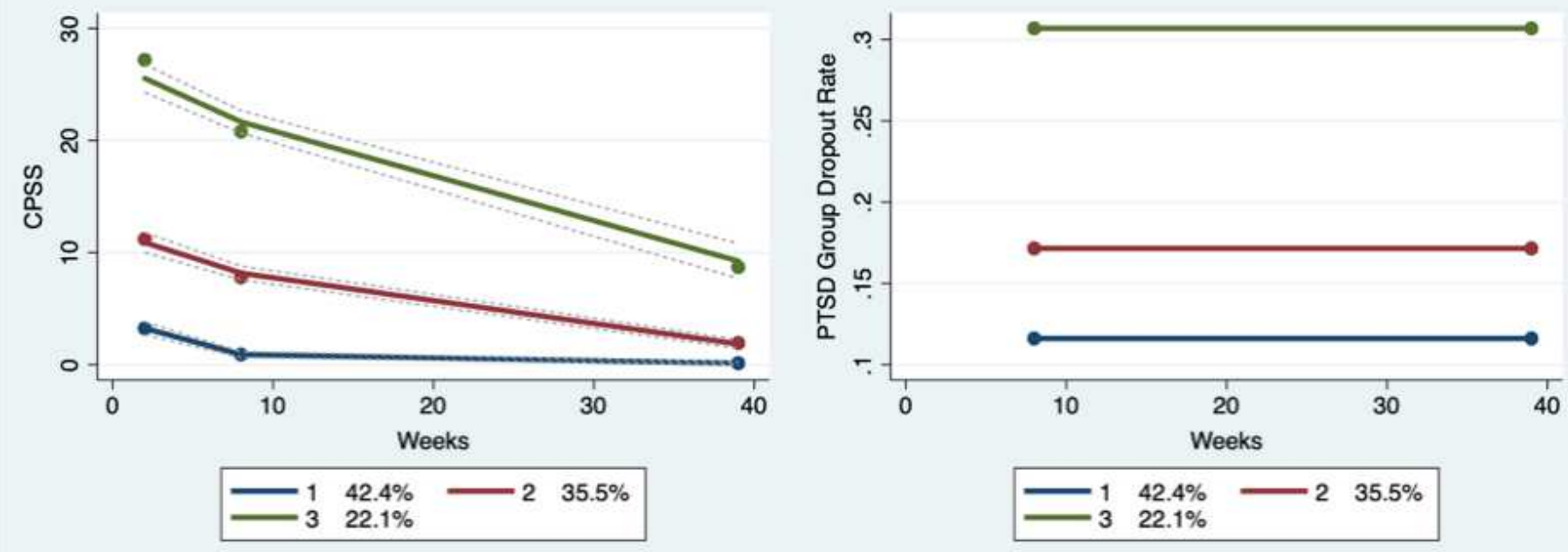

Figure 2a Final trajectory and dropout model of SPSS 
Trajectories of Post-traumatic Stress and Depression Following Accident: Course and Risk Factors
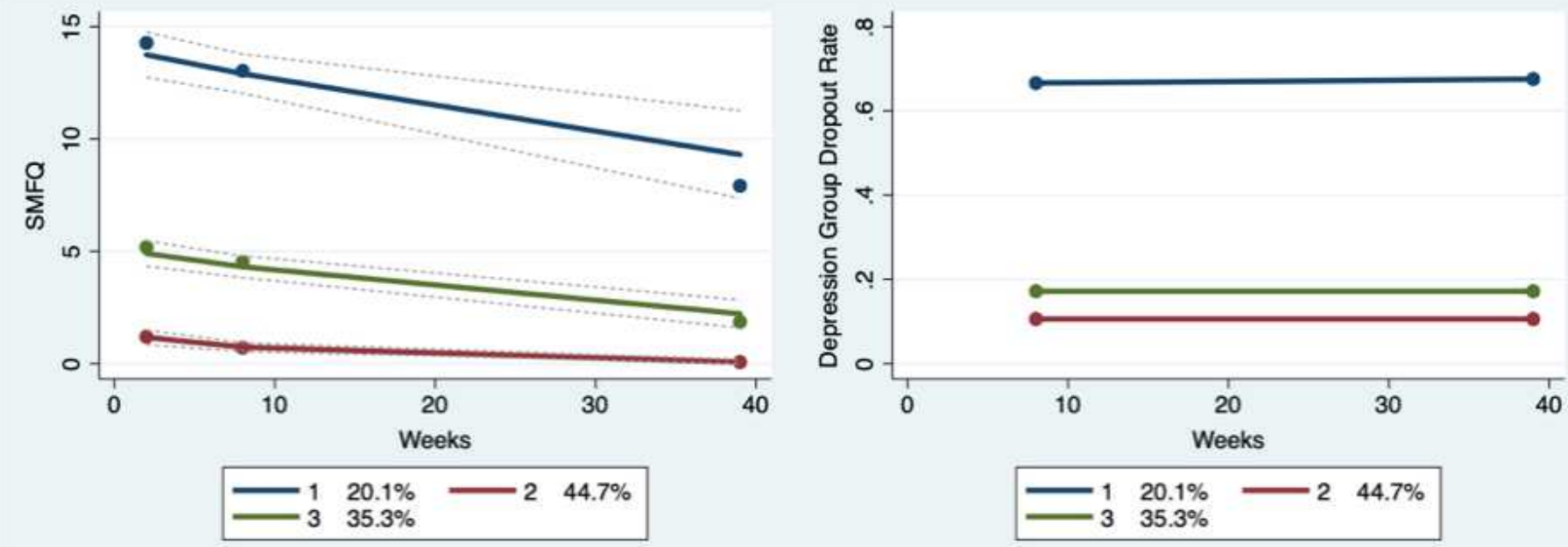

Figure 2b. Final trajectory and dropout model of SMFQ.

\section{(a): Probability of Depression group conditional on PTSD group}

- Low Depression $\quad$ Medium Depression $\quad$ High Depression

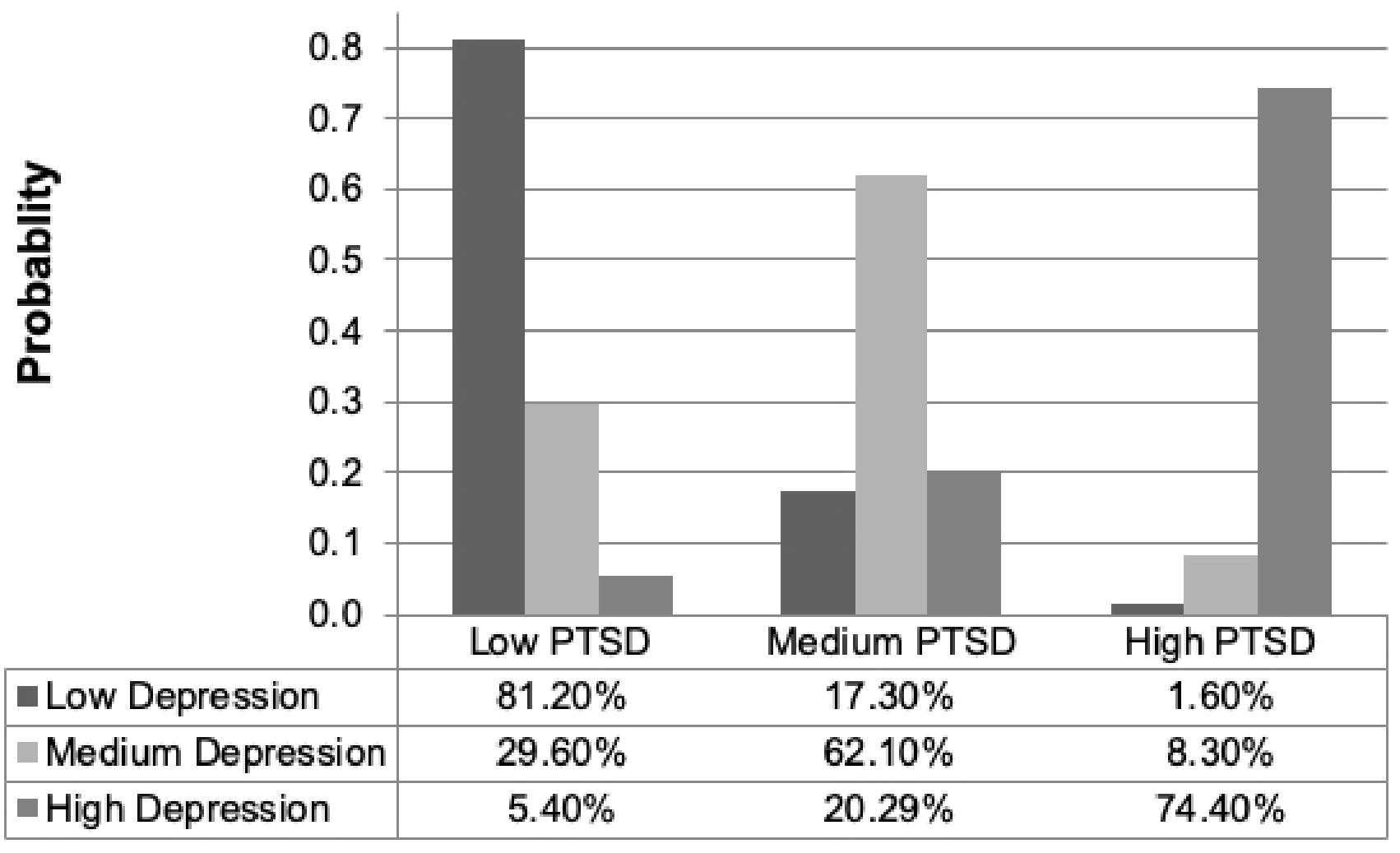

Figure 3a: Conditional probability of depression if PTSD is known 


\section{(b): Probability of PTSD group conditional on depression group}

घ Low PTSD $\quad$ Medium PTSD $\quad$ High PTSD

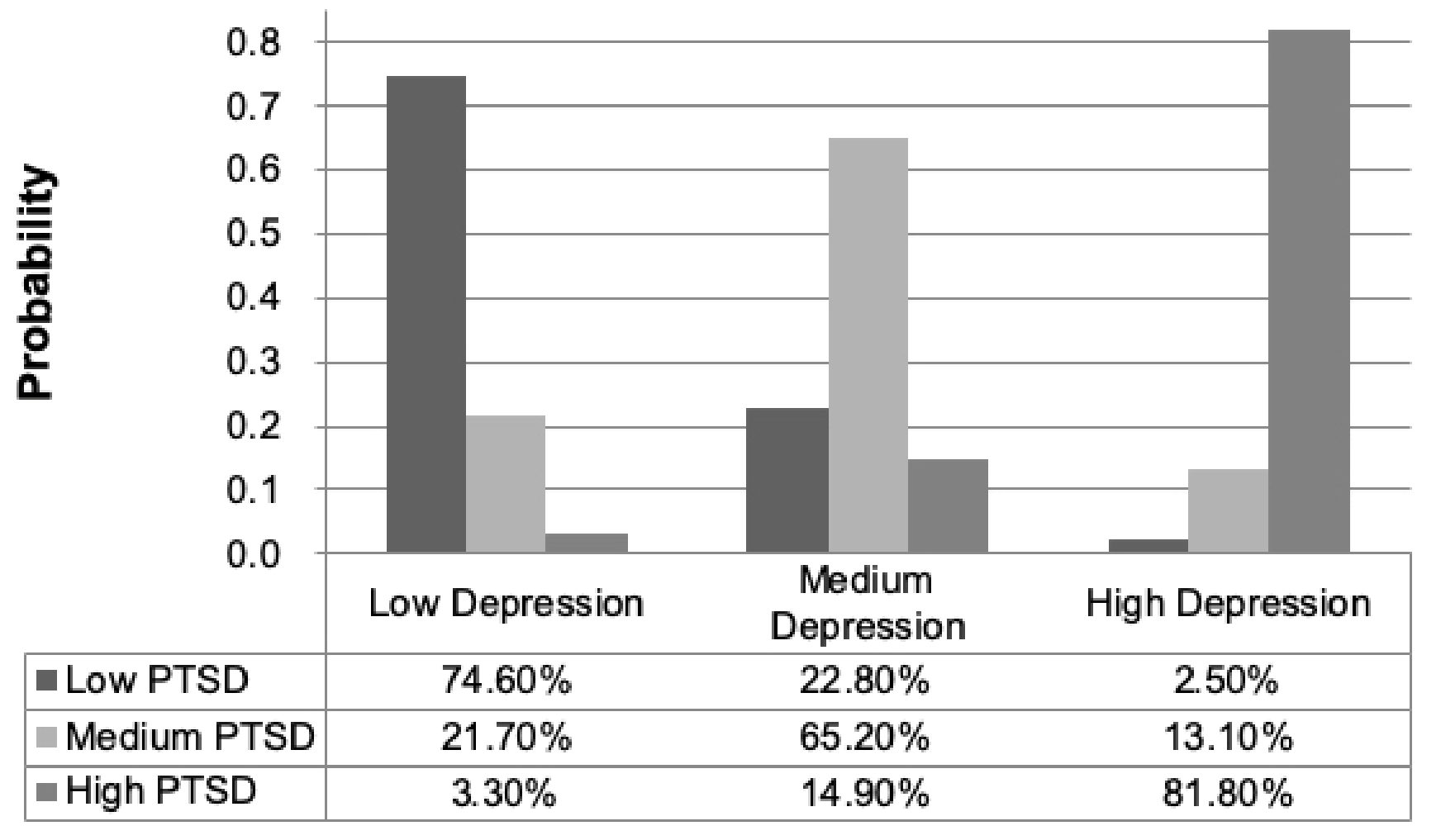

Figure 3b: Conditional probability of PTSD if depression is known 
Trajectories of Post-traumatic Stress and Depression Following Accident: Course and Risk Factors

\begin{tabular}{|c|c|c|c|c|c|}
\hline \multicolumn{6}{|c|}{ 1a: PTSD trajectory groups } \\
\hline & Group & Mean & Est. Mean & $95 \% \mathrm{Cl}$ & Dropout $\%$ \\
\hline \multirow{3}{*}{2 weeks } & Low & 3.243 & 3.238 & {$[2.703,3.773]$} & - \\
\hline & Medium & 11.125 & 10.873 & {$[9.987,11.759]$} & - \\
\hline & High & 27.582 & 25.789 & {$[24.529,27.048]$} & - \\
\hline \multirow{4}{*}{8 weeks } & Low & .879 & .875 & {$[.607,1.142]$} & $14.1 \%$ \\
\hline & Medium & 7.780 & 8.096 & {$[7.498,8.695]$} & $15.5 \%$ \\
\hline & High & 20.824 & 21.746 & {$[20.742,22.750]$} & $30.2 \%$ \\
\hline & Low & .127 & .127 & {$[0, .258]$} & $14.1 \%$ \\
\hline \multirow[t]{2}{*}{9 months } & Medium & 1.921 & 1.854 & {$[1.467,2.241]$} & $15.5 \%$ \\
\hline & High & 8.709 & 9.271 & {$[7.718,10.825]$} & $30.2 \%$ \\
\hline \multicolumn{6}{|c|}{ 1b: Depression trajectory groups } \\
\hline \multirow{4}{*}{2 weeks } & Group & Mean & Est. Mean & $95 \% \mathrm{Cl}$ & Dropout\% \\
\hline & Low & 1.230 & 1.231 & {$[.9152,1.546]$} & - \\
\hline & Medium & 5.220 & 4.998 & {$[4.438,5.559]$} & - \\
\hline & High & 14.351 & 13.831 & {$[12.762,14.899]$} & - \\
\hline \multirow{3}{*}{8 weeks } & Low & .702 & .753 & {$[.5677, .938]$} & $16.3 \%$ \\
\hline & Medium & 4.599 & 4.375 & {$[3.898,4.852]$} & $11.9 \%$ \\
\hline & High & 12.936 & 12.933 & {$[12.021,13.845]$} & $65.1 \%$ \\
\hline \multirow{3}{*}{9 months } & Low & .068 & .0645 & {$[0, .185]$} & $16.3 \%$ \\
\hline & Medium & 1.848 & 2.248 & {$[1.625,2.871]$} & $11.9 \%$ \\
\hline & High & 7.960 & 9.246 & {$[7.321,11.171]$} & $65.1 \%$ \\
\hline
\end{tabular}

1c: Joint probability of combined membership

Depression Group

\begin{tabular}{lllll} 
& & & Medium & High \\
\cline { 3 - 5 } & Low & $34.5 \%$ & $7.3 \%$ & $0.7 \%$ \\
PTSD Group & Medium & $10.5 \%$ & $22.1 \%$ & $3.0 \%$ \\
& High & $1.2 \%$ & $4.4 \%$ & $16.3 \%$ \\
\hline
\end{tabular}


Trajectories of Post-traumatic Stress and Depression Following Accident: Course and Risk Factors

Table 2:

Multinomial logistic regression analysis

2a: Predictors of high PTSD group using low PTSD symptom group as referent

\begin{tabular}{|c|c|c|c|c|c|}
\hline \multicolumn{3}{|c|}{ Log likelihood = - 112. 91091} & \multicolumn{3}{|c|}{$\begin{array}{l}\text { \# of obs: } 214 \\
\text { LR chi2 }(20)=228.84 \\
\text { Prob }>\text { chi2 }=0.000 \\
\text { Psudo } R 2=0.5033\end{array}$} \\
\hline & 'RRR & Std. Err. & $\mathbf{Z}$ & $\mathbf{P}>|\mathrm{Z}|$ & [95\% Conf. Interval] \\
\hline Age & 1.66 & 1.31 & 0.64 & 0.520 & {$[.36,7.73]$} \\
\hline Gender & 1.05 & .14 & 0.38 & 0.704 & {$[.81,1.37]$} \\
\hline $\begin{array}{l}\text { Pre-trauma } \\
\text { emotional wellbeing }\end{array}$ & .92 & .09 & -0.93 & 0.354 & {$[.76,1.10]$} \\
\hline $\begin{array}{l}\text { Peri-trauma panic }{ }^{*} \\
\text { Post-trauma anxiety }\end{array}$ & $\begin{array}{l}2.09 \\
1.07\end{array}$ & $\begin{array}{l}.45 \\
.05\end{array}$ & $\begin{array}{l}3.47 \\
1.33\end{array}$ & $\begin{array}{l}0.001 \\
0.182\end{array}$ & $\begin{array}{l}{[1.38,3.19]} \\
{[.97,1.17]}\end{array}$ \\
\hline $\begin{array}{l}\text { Cognitive } \\
\text { apprasial }^{\star}\end{array}$ & 1.26 & .08 & 3.86 & 0.000 & {$[1.12,1.42]$} \\
\hline Rumination* & 1.61 & .33 & 2.32 & 0.020 & {$[1.08,2.39]$} \\
\hline $\begin{array}{l}\text { Thought } \\
\text { suppression* }\end{array}$ & 1.27 & .13 & 2.35 & 0.019 & {$[1.04,1.56]$} \\
\hline $\begin{array}{l}\text { Adaptive processing } \\
\text { Self blame } \\
\text { cons }\end{array}$ & $\begin{array}{l}.85 \\
.68 \\
6.34 \mathrm{e}-09\end{array}$ & $\begin{array}{l}.09 \\
.14 \\
2.42 \mathrm{e}-08\end{array}$ & $\begin{array}{l}-1.47 \\
-1.83 \\
-4.94\end{array}$ & $\begin{array}{l}0.140 \\
0.068 \\
0.000\end{array}$ & $\begin{array}{l}{[.68,1.06]} \\
{[.45,1.03]} \\
{[3.54 \mathrm{e}-12, .0000114]}\end{array}$ \\
\hline
\end{tabular}

2b: Predictors of high depression group using low depression symptom group as referent

\begin{tabular}{|c|c|c|c|c|c|}
\hline \multicolumn{2}{|c|}{ Log likelihood $=-110.86479$} & \multirow[b]{2}{*}{ Std. Err. } & \multicolumn{2}{|c|}{$\begin{array}{l}\text { \# of obs: } 214 \\
\text { LR chi2 } 2(20)=225.83 \\
\text { Prob }>\text { chi2 }=0.000 \\
\text { Psudo } R 2=0.5046\end{array}$} & \multirow[b]{2}{*}{ [95\% Conf. Interval] } \\
\hline & 'RRR & & $\mathbf{Z}$ & $\mathbf{P}>|\mathbf{Z}|$ & \\
\hline Gender & .95 & .74 & 0.07 & 0.947 & {$[.21,4.34]$} \\
\hline Age & 1.30 & .18 & 1.89 & 0.059 & {$[.99,1.71]$} \\
\hline $\begin{array}{l}\text { Pre-trauma } \\
\text { emotional } \\
\text { wellbeing }^{\star}\end{array}$ & 1.24 & .12 & 2.24 & 0.025 & {$[1.03,1.49]$} \\
\hline Peri-trauma panic & .98 & .18 & -0.09 & 0.930 & {$[.68,1.42]$} \\
\hline $\begin{array}{l}\text { Post-trauma } \\
\text { anxiety* }\end{array}$ & 1.21 & .06 & 3.62 & 0.000 & {$[1.09,1.34]$} \\
\hline $\begin{array}{l}\text { Cognitive } \\
\text { apprasial* }^{\star}\end{array}$ & 1.31 & .08 & 4.22 & 0.000 & {$[1.15,1.48]$} \\
\hline Rumination & 1.28 & .24 & 1.30 & 0.194 & {$[.88,1.86]$} \\
\hline Thought suppression & 1.06 & .12 & 0.61 & 0.542 & {$[.87,1.30]$} \\
\hline Adaptive processing & .87 & .09 & -1.20 & 0.229 & {$[.70,1.09]$} \\
\hline Self blame & 1.34 & .25 & 1.54 & 0.123 & {$[.92,1.93]$} \\
\hline _cons & $2.30 \mathrm{e}-13$ & $1.05 e-12$ & -6.38 & 0.000 & $\begin{array}{l}{[3.03 e-17 \quad 1.75 e-} \\
09]\end{array}$ \\
\hline
\end{tabular}




\begin{tabular}{|c|c|c|}
\hline Scale & Items & Responses \\
\hline $\begin{array}{l}\text { Post-trauma } \\
\text { dissociation }\end{array}$ & $\begin{array}{l}\text { 1. Being so shocked that I didn't feel anything } \\
\text { 2. Not really noticing what was going on around me (like being in a bubble) } \\
\text { 3. Feeling like I was in a dream } \\
\text { 4. Feeling like I was outside my body, or that my body didn't belong to me }\end{array}$ & $\begin{array}{l}\text { Not at all or only one time, Once a } \\
\text { week or less/once in a while, } 2 \text { to } \\
4 \text { times a week/half the time, } 5 \text { or } \\
\text { more times a week/almost always } \\
(0,1,2,3)\end{array}$ \\
\hline Rumination & $\begin{array}{l}\text { 1. I keep wishing that I could go back in time and stop the event from happening. } \\
\text { 2. Whenever I think of the event I wonder why it happened to us. } \\
\text { 3. I am always wondering if my family or I might get hurt again. }\end{array}$ & $\begin{array}{l}\text { Disagree a lot, Disagree a bit, } \\
\text { Agree a bit, Agree a, lot }(1,2,3, \\
\text { 4) }\end{array}$ \\
\hline $\begin{array}{l}\text { CTSQ: thought } \\
\text { suppression }\end{array}$ & $\begin{array}{l}\text { 1. When I have thoughts or memories about what happened I try hard to push } \\
\text { them out of my mind. } \\
\text { 2. When I have thoughts or memories about what happened I try to think about } \\
\text { something else instead. } \\
\text { 3. When I have thoughts or memories about what happened I keep busy doing } \\
\text { other things } \\
\text { 4. When I have thoughts or memories about what happened I try to control how I } \\
\text { feel. } \\
5 \text {. When I have thoughts or memories about what happened I try to get rid of the } \\
\text { memory. }\end{array}$ & $\begin{array}{l}\text { Disagree a lot, Disagree a bit, } \\
\text { Agree a bit, Agree a, lot }(1,2,3 \text {, } \\
\text { 4) }\end{array}$ \\
\hline $\begin{array}{l}\text { CAPQ: adaptive } \\
\text { processing }\end{array}$ & $\begin{array}{l}\text { 1. I try to understand what happened during the event. } 2 . \text { I try to piece it together in } \\
\text { my mind. } 3 \text {. I explain the whole story to my friends and family. } 4 \text {. I think it through } \\
\text { so that what happened makes sense in my mind. } 5.1 \text { get help }\end{array}$ & $\begin{array}{l}\text { Disagree a lot, Disagree a bit, } \\
\text { Agree a bit, Agree a, lot }(1,2,3 \text {, } \\
\text { 4) }\end{array}$ \\
\hline Self-blaming & $\begin{array}{l}\text { 1. I made the event happen. } \\
\text { 2. It was my fault the event happened. }\end{array}$ & $\begin{array}{l}\text { Disagree a lot, Disagree a bit, } \\
\text { Agree a bit, Agree a, lot }(1,2,3 \text {, } \\
\text { 4) }\end{array}$ \\
\hline $\begin{array}{l}\text { CPAS: pre-trauma } \\
\text { emotional wellbeing }\end{array}$ & $\begin{array}{l}1 \text { Before the event I used to feel really sad sometimes. } \\
2 \text { Before the event I sometimes felt very nervous or scared about things. } \\
3 \text { Before the event I had big problems with feeling very sad or scared. } \\
4 \text { Before the event I always knew someone I could eally trust. } \\
5 \text { Before the event there were always people in my life who helped me. } \\
6 \text { Before the event I could always talk to my family and friends about my thoughts } \\
\text { and feelings. } \\
7 \text { Before the event I always had good support from my friends and my family. } \\
8 \text { Before the event I often got angry at people. } \\
9 \text { Before the event I often got upset and scared. } \\
10 \text { Before the event I was very calm and relaxed. }\end{array}$ & $\begin{array}{l}\text { Not at all like that, } \text { A little bit like } \\
\text { that, Like that, Very much like that } \\
(1,2,3,4)\end{array}$ \\
\hline $\begin{array}{l}\text { CPP: peri-traumatic } \\
\text { panic }\end{array}$ & $\begin{array}{l}\text { 1. did you have pain in your chest? } \\
\text { 2. did you feel very hot or cold? } \\
\text { 3. did you feel like you were choking, like you couldn't breathe? } \\
\text { 4. did you feel dizzy or faint? } \\
\text { 5. did you feel like you couldn't control yourself anymore, or that you were going } \\
\text { mad? } \\
\text { 6. did you heart beat very fast, or go boom-boom-boom very loud? } \\
\text { 7. did you feel sick, like you might throw up? } \\
\text { 8. did you sweat a lot? } \\
\text { 9. did you find it hard to breathe? } \\
\text { 10. did you shake or tremble? }\end{array}$ & No, Yes $(0,1)$ \\
\hline $\begin{array}{l}\text { CPTCl: cognitive } \\
\text { appraisal }\end{array}$ & $\begin{array}{l}\text { 1. Anyone could hurt me. } \\
\text { 2. Everyone lets me down. } \\
\text { 3. I am a coward. } \\
\text { 4.My reactions since the frightening event mean I have changed for the worse. } \\
\text { 5. I don't trust people. } \\
\text { 6. My reactions since the frightening event mean something is seriously wrong with } \\
\text { me. } \\
\text { 7. I am no good. } \\
\text { 8. Not being able to get over all my fears means that I am a failure. } \\
\text { 9. Small things upset me. } \\
\text { 10. I can't cope when things get tough. } \\
\text { 11. I can't stop bad things from happening to me. } \\
\text { 12. I have to watch out for danger all the time. } \\
\text { 13. My reactions since the frightening event mean I will never get over it. } \\
\text { 14. I used to be a happy person but now I am always sad. } \\
\text { 15. Bad things always happen. } \\
\text { 16. I will never be able to have normal feelings again. } \\
\text { 17.I'm scared that l'll get so angry that l'll break something or hurt someone. } \\
\text { 18. Life is not fair. } \\
\text { 19. My life has been destroyed by the frightening event. } \\
\text { 20. I feel like I am a different person since the frightening event. } \\
\text { 21. My reactions since the frightening event show that I must be going crazy. } \\
\text { 22. Nothing good can happen to me anymore. } \\
\text { 23. Something terrible will happen if I do not try to control my thoughts about the } \\
\text { frightening event. } \\
\text { 24. The frightening event has changed me forever. } \\
\text { 25. I have to be really careful because something bad could happen. }\end{array}$ & $\begin{array}{l}\text { Don't agree at all, Don't agree a } \\
\text { bit, Agree a bit, Agree a lot } \\
(1,2,3,4)\end{array}$ \\
\hline
\end{tabular}


Trajectories of Post-traumatic Stress and Depression Following Accident: Course and Risk Factors

Appendix B: Distribution Estimation R Scripts and Results

\# load libraries
library(MASS)

R Script

library(survival)

library(fitdistrplus)

\# read data

tr <- read.csv("Traj217.csv", header = TRUE, sep = ",", quote = "l"", dec = ".", fill = TRUE)

w2cpss = tr[, "w2 CPSSTotal"]

w2smfq = tr[, "w2_SMFQTotal"]

\# check if the distributions are exponential

fw2cpss <- fitdist(w2cpss, "exp", method="mle")

fw2smfq <-fitdist(w2smfq, "exp", method="mle")

\# display summary

summary(fw2cpss)

summary(fw2smfq)

\# plot the result

plot(fw2cpss)

plot(fw2smfq)

Fitting of the distribution ' exp ' by maximum likelihood

R Output

Parameters :

estimate Std. Error

rate 0.087914320 .00596724

Loglikelihood: -744.6122 AIC: 1491.224 BIC: 1494.604

Fitting of the distribution ' exp ' by maximum likelihood

Parameters :

estimate Std. Error

rate 0.1913580 .01298987

Loglikelihood: -575.8332 AIC: 1153.666 BIC: 1157.046 
Trajectories of Post-traumatic Stress and Depression Following Accident: Course and Risk Factors

Empirical and theoretical dens.

Q-Q plot
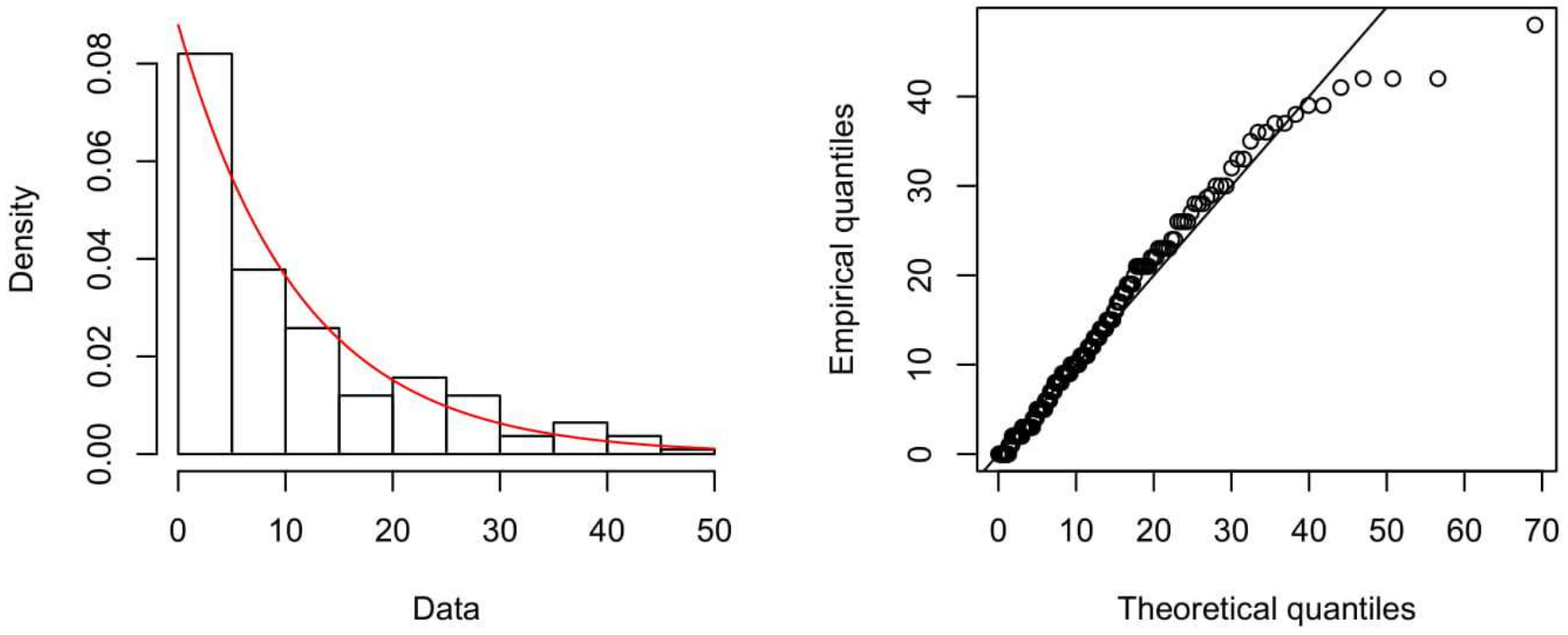

Empirical and theoretical CDFs

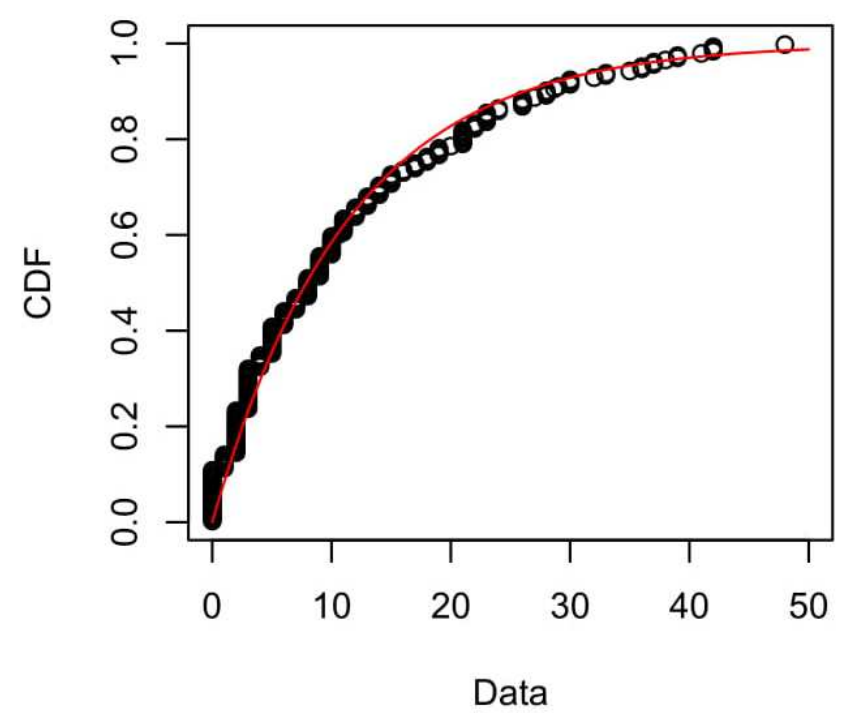

P.P plot

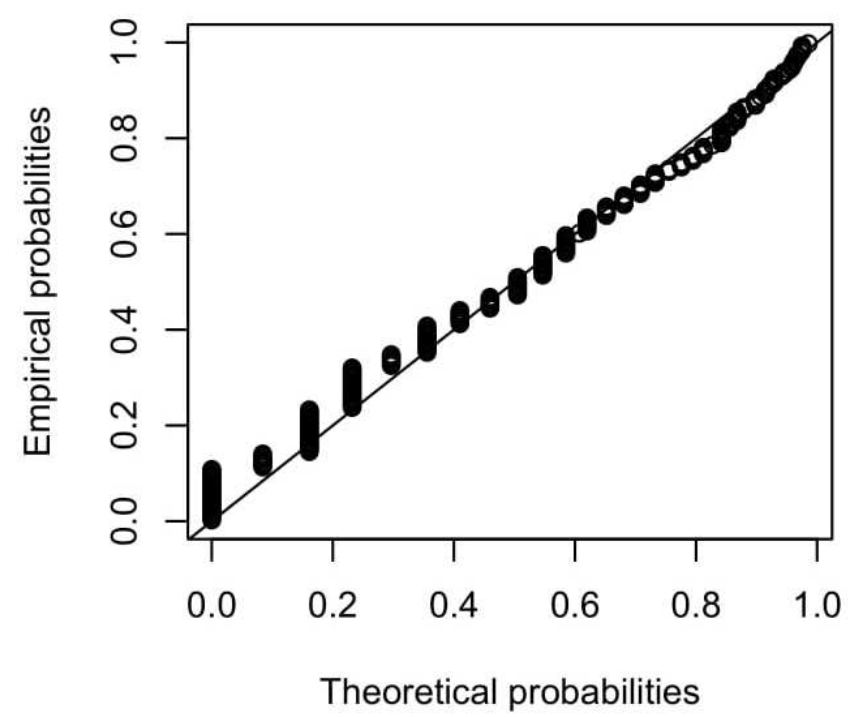

CPSS score at week 2 distribution estimation - fitness to exponential distribution 
Trajectories of Post-traumatic Stress and Depression Following Accident: Course and Risk Factors

Empirical and theoretical dens.

Q-Q plot
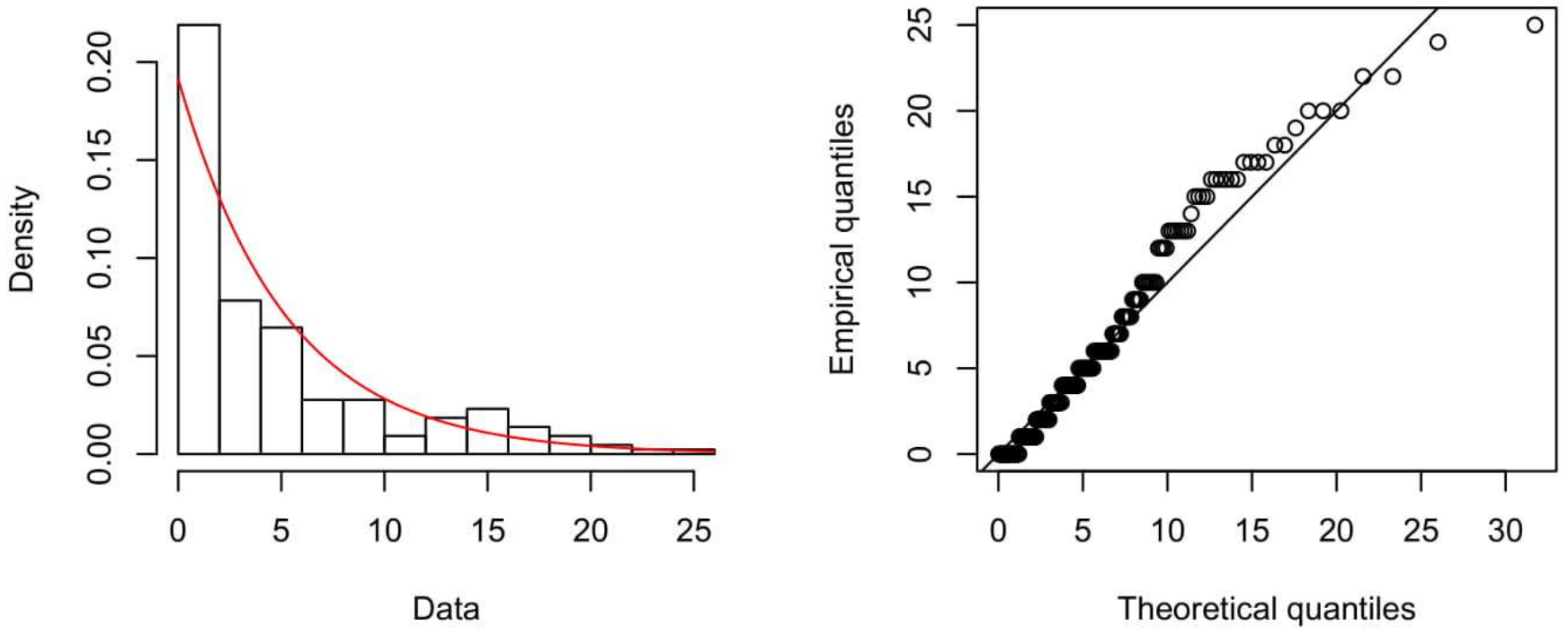

Empirical and theoretical CDFs
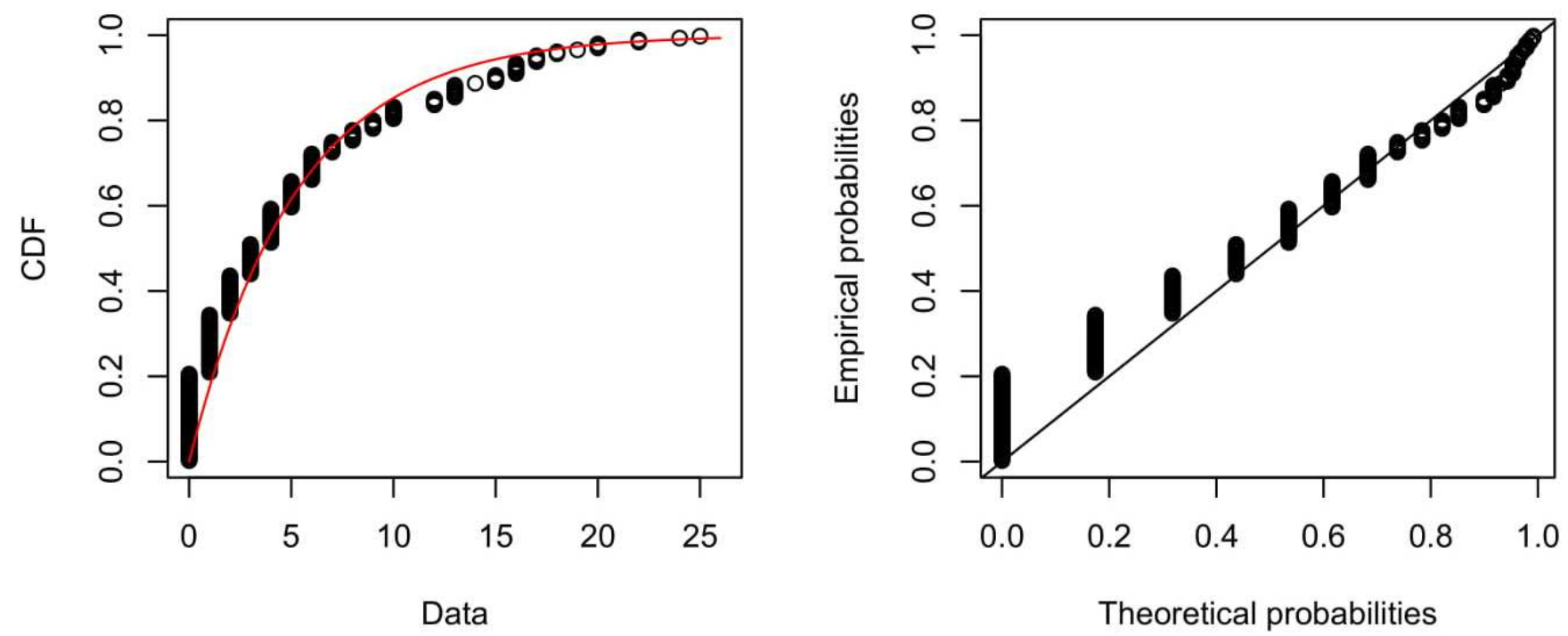

SMPQ score at week 2 distribution estimation - fitness to exponential distribution 
Trajectories of Post-traumatic Stress and Depression Following Accident: Course and Risk Factors

\section{Appendix C: STATA Script for Single and Joint Trajectory Modeling}

//Single modeling for PTSD, 3 groups

traj, var(cpss1-cpss3) indep(t1-t3) model(zip) order(2 111$)$ iorder (-1 -1 0) dropout (1 111$)$

//Single modeling for depression, 3 groups`

traj, var(smfq1-smfq3) indep(t1-t3) model(zip) order(1 111$)$ iorder $\left(\begin{array}{lll}0 & 0 & 0\end{array}\right)$ dropout $\left(\begin{array}{lll}1 & 1 & 1\end{array}\right)$

${ }^{*}$ Joint modelling*/

//First run, probe starting values

traj, var(cpss1-cpss3) indep(t1-t3) model(zip) order(2 1 1) iorder (-1 -1 0) dropout1 (1 1 0) var2(smfq1-smfq3) indep2(t1-t3) model2(zip) order2(1 1

1) iorder2 ( $\left.\begin{array}{lll}0 & 1 & 0\end{array}\right)$ dropout2 $\left(\begin{array}{lll}1 & 1 & 1\end{array}\right)$ nderiv detail

// Starting parameters attained from last step

matrix sv $=1.67953, \quad-0.26089, \quad 0.00429, \quad 2.48455, \quad-0.04914, \quad 3.36248, \quad-0.02842,-1.80910, \quad-1.69506, \quad-0.83801, \quad-2.85973$,

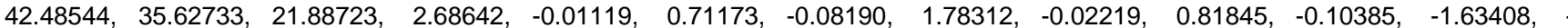

1.99887, -3.27396, -0.90256, -1.97780, 1.54817, 81.15344, 17.29839, 8.29685, 29.60195, 62.10119, 74.36043, 5.35081,

20.28876

//Joint modelling with starting parameters

traj, var(cpss1-cpss3) indep(t1-t3) model(zip) order(2 1 1) iorder (-1 -1 0) dropout1 (0 0 0) var2(smfq1-smfq3) indep2(t1-t3) model2(zip) order2(1 1

1) iorder2 $\left(\begin{array}{lll}0 & 0 & 0\end{array}\right)$ dropout2 $\left(\begin{array}{lll}1 & 0 & 0\end{array}\right)$ nderiv start(sv)

matrix list e(plot1) //retrieve PTSD group parameters

matrix list e(plot2) //retrieve depression group parameters

trajplot, ytitle(CPSS) xtitle(Weeks) ci //plot PTSD trajectories

trajplot, ytitle(PTSD Group Dropout Rate) xtitle(Weeks) dropout //plot PTSD dropout

trajplot, model(2) ytitle(SMFQ) xtitle(Weeks) ci //plot depression trajectories

trajplot, model(2) ytitle(Depression Group Dropout Rate) xtitle(Weeks) dropout //plot depression dropout 
Trajectories of Post-traumatic Stress and Depression Following Accident: Course and Risk Factors

Appendix D: STATA Script for Multinomial Logistic Regression Analysis

//PTSD predictor analysis

mlogit_traj_ptsd_group gender age w2_cpastotal w2_cpptotal w2_scastotal w2_cptcitotal w2_rumination w2_thoughtsuppression w2_adaptiveprocessing w2_selfblame, $\mathrm{rrr}$

//Depression predictor analysis

mlogit traj_dep_group gender age w2_cpastotal w2_cpptotal w2_scastotal w2_cptcitotal w2_rumination w2_thoughtsuppression w2_adaptiveprocessing w2_selfblame, rrr 
Trajectories of Post-traumatic Stress and Depression Following Accident: Course and Risk Factors

Supplementary Tables

Table S1:

$B I C$ values and comparisons of the models

\begin{tabular}{|c|c|c|c|c|c|}
\hline \multirow[b]{2}{*}{$\begin{array}{c}\text { \# of groups in } \\
\text { Model }\end{array}$} & \multirow[b]{2}{*}{ Null Model } & \multicolumn{2}{|c|}{ PTSD Models } & \multicolumn{2}{|c|}{ Depression Models } \\
\hline & & BIC & $\begin{array}{l}\operatorname{2log}_{e}\left(B_{10}\right) \\
\approx 2(\Delta B I C)\end{array}$ & BIC & $\begin{array}{l}2 \log _{e}\left(B_{10}\right) \\
\approx 2(\Delta B I C)\end{array}$ \\
\hline 2 & & -2038.52 & & -1526.07 & \\
\hline 3 & 2 & -1951.57 & 173.9 & -1476.44 & 99.26 \\
\hline 4 & 3 & -1882.63 & 137.88 & -1473.23 & 6.42 \\
\hline
\end{tabular}

\section{Table S2:}

Output of joint model of PTSD symptoms and depression symptoms

Maximum Likelihood Estimates:

Model 1: Zero Inflated Poisson (zip)

Model 1: Zero Inflated Poisson (zip)

Standard T for HO:

\begin{tabular}{llllll}
\hline Group & Parameter & Estimate & Error & Parameter $=\mathbf{0}$ & Prob > $\mathbf{T} \mid$ \\
\hline $\mathbf{1}$ & Intercept & 1.67953 & 0.12693 & 13.232 & 0.0000 \\
& Linear & -0.26089 & 0.03594 & 7.259 & 0.0000 \\
$\mathbf{2}$ & Quadratic & 0.00427 & 0.00092 & 4.625 & 0.0000 \\
& Intercept & 2.48455 & 0.04472 & 55.557 & 0.0000 \\
$\mathbf{3}$ & Linear & -0.04914 & 0.00326 & -15.082 & 0.0000 \\
& Intercept & 3.36248 & 0.02731 & 123.136 & 0.0000 \\
$\mathbf{1}$ & Linear & -0.02842 & 0.00256 & -11.091 & 0.0000 \\
$\mathbf{2}$ & Drop0 & -1.80910 & 0.22638 & -7.992 & 0.0000 \\
$\mathbf{3}$ & Drop0 & -1.69506 & 0.23719 & -7.146 & 0.0000 \\
& Drop0 & -0.83801 & 0.23408 & -3.580 & 0.0004 \\
$\mathbf{3}$ & Alpha0 & & & & 0.0000 \\
& & -2.85973 & 0.49412 & -5.787 & \\
\hline Group membership & & & & \\
\hline $\mathbf{2}$ & $(\%)$ & 42.48544 & 3.75631 & 11.310 & 0.0000 \\
$\mathbf{3}$ & $(\%)$ & 21.62733 & 3.61810 & 9.847 & 0.0000 \\
\end{tabular}

Model 2: Zero Inflated Poisson (zip)

\begin{tabular}{|c|c|c|c|c|c|}
\hline Group & Parameter & Estimate & Error & Parameter $=0$ & Prob $>|\mathrm{T}|$ \\
\hline \multirow[t]{2}{*}{1} & Intercept & 2.68642 & 0.04251 & 63.202 & 0.0000 \\
\hline & Linear & -0.01119 & 0.00323 & -3.460 & 0.0006 \\
\hline \multirow[t]{2}{*}{2} & Intercept & 0.71173 & 0.15823 & 4.498 & 0.0000 \\
\hline & Linear & -0.08190 & 0.01455 & -5.630 & 0.0000 \\
\hline \multirow[t]{2}{*}{3} & Intercept & 1.78312 & 0.05865 & 30.401 & 0.0000 \\
\hline & Linear & -0.02220 & 0.00411 & -5.404 & 0.0000 \\
\hline \multirow[t]{2}{*}{1} & Drop0 & 0.81845 & 0.47479 & 1.724 & 0.0850 \\
\hline & Drop1 & -0.10385 & 0.03306 & -3.141 & 0.0017 \\
\hline 2 & Drop0 & -1.63408 & 0.19487 & -8.385 & 0.0000 \\
\hline 3 & Drop0 & -1.99887 & 0.23574 & -8.479 & 0.0000 \\
\hline 1 & Alpha0 & -3.27396 & 0.63149 & -5.184 & 0.0000 \\
\hline 2 & Alpha0 & -0.90256 & 0.29765 & -3.032 & 0.0025 \\
\hline 3 & Alpha0 & -1.97780 & 0.30100 & -6.571 & 0.0000 \\
\hline \multicolumn{6}{|c|}{ Group membership (model 2 group model 1 group) } \\
\hline $1 \mid 1$ & $(\%)$ & 1.54817 & 1.78252 & 0.869 & 0.3853 \\
\hline $2 \mid 1$ & (\%) & 81.15344 & 5.01356 & 16.187 & 0.0000 \\
\hline $3 \mid 1$ & (\%) & 17.29839 & 4.91291 & 3.521 & 0.0004 \\
\hline $1 \mid 2$ & $(\%)$ & 8.29686 & 3.81094 & 2.177 & 0.0297 \\
\hline $2 \mid 2$ & (\%) & 29.60195 & 6.58519 & 4.495 & 0.0000 \\
\hline $3 \mid 2$ & (\%) & 62.10119 & 6.82835 & 9.095 & 0.0000 \\
\hline $1 \mid 3$ & (\%) & 74.36043 & 6.80319 & 10.930 & 0.0000 \\
\hline $2 \mid 3$ & (\%) & 5.35081 & 3.84131 & 1.393 & 0.1639 \\
\hline $3 \mid 3$ & (\%) & 20.28876 & 6.54372 & 3.100 & 0.0020 \\
\hline
\end{tabular}

Group membership (model 2 group)*

\begin{tabular}{ll}
\multicolumn{2}{l}{ Group membership (model 2 group) } \\
\hline $\mathbf{1}$ & $(19.9 \%)$ \\
$\mathbf{2}$ & $(46.2 \%)$ \\
$\mathbf{3}$ & $(33.9 \%)$ \\
\hline
\end{tabular}

$\mathrm{BIC}=-3612.74(\mathrm{~N}=1125) \quad \mathrm{BIC}=-3586.41(\mathrm{~N}=217) \quad \mathrm{AIC}=-3532.33 \quad \mathrm{II}=-3500.33$

*the group membership of model 2 was calculated based on the estimated group membership of model 1 and the conditional group membership according to the law of probability 
Trajectories of Post-traumatic Stress and Depression Following Accident: Course and Risk Factors Output of regression analysis between trauma severity and SMFQ at 2 weeks (T1), 2 months (T2) and 9 months (T3)

\begin{tabular}{|c|c|c|c|c|c|}
\hline & Trauam severity & Coef. & Std. Err. & $\mathbf{T}$ & $P>|t|$ \\
\hline \multirow[t]{4}{*}{ T1 } & number of injuries & .0053119 & .0039664 & 1.34 & 0.182 \\
\hline & fracture & -.005303 & .0049448 & -1.07 & 0.285 \\
\hline & resus & -.0092355 & .0037676 & -2.45 & 0.045 \\
\hline & permanent loss of function & -.0080803 & .0053285 & -1.52 & 0.131 \\
\hline \multirow[t]{4}{*}{ T2 } & number of injuries & .0015232 & .0043968 & 0.35 & 0.729 \\
\hline & fracture & -.0089812 & .0055062 & -1.63 & 0.104 \\
\hline & resus & -.0086361 & .0041046 & -2.10 & 0.177 \\
\hline & permanent loss of function & -.0129129 & .0058679 & -2.20 & 0.069 \\
\hline \multirow[t]{4}{*}{ T3 } & number of injuries & .0093984 & .0084193 & 1.12 & 0.266 \\
\hline & fracture & .0089996 & .0116276 & 0.77 & 0.440 \\
\hline & resus & -.0111198 & .0087955 & -1.26 & 0.208 \\
\hline & permanent loss of function & -.0073347 & .0127083 & -0.58 & 0.565 \\
\hline
\end{tabular}

Note: zero-order correlations were carried out seperately without any controls 